Article

\title{
The Energy-Efficient Operation Problem of a Freight Train Considering Long-Distance Steep Downhill Sections
}

\author{
Xuan Lin ${ }^{1}$, Qingyuan Wang ${ }^{1, *}$, Pengling Wang ${ }^{2}$, Pengfei Sun ${ }^{1}$ and Xiaoyun Feng ${ }^{1}$ \\ 1 School of Electrical Engineering, Southwest Jiaotong University, Chengdu 610031, China; \\ linstu@163.com (X.L.); spf0325@163.com (P.S.); fengxy@home.swjtu.edu.cn (X.F.) \\ 2 Department of Transport and Planning, Delft University of Technology, \\ Mekelweg 2, 2628 CD Delft, The Netherlands; p.l.wang@tudelft.nl \\ * Correspondence: wangqy@home.swjtu.edu.cn; Tel.: +86-134-5854-8923 \\ Academic Editor: Carl-Fredrik Lindberg \\ Received: 13 April 2017; Accepted: 5 June 2017; Published: 10 June 2017
}

\begin{abstract}
With the energy consumption rising in rail transport, the railway sector is showing increasing interest in the energy-efficient operation of freight trains. Freight trains require more complicated driving strategies than ordinary passenger trains do due to their heavy loads, especially in the long-distance steep downhill (LDSD) sections that are very common in freight rail lines in China. This paper studies the energy-efficient operation of a freight train considering LDSD sections. An optimal control model including regenerative and pneumatic braking is developed for the freight train. Then, when a train leaves/enters the LDSD section, we verify the uniqueness of control transitions and discuss the speed profile linkage between LDSD and its adjacent sections, which indicates that the periodic braking should be applied on LDSD sections for optimality. Additionally, given the same running time for the entire journey, our analysis shows that electrical braking-full braking strategy is more energy-efficient than coasting-full braking strategy on LDSD sections. Finally, a numerical algorithm for the optimal driving solution is proposed. The simulation results demonstrate that the driving strategies generated by the proposed algorithm performs better than those from fuzzy predictive control and field operation regarding energy saving.
\end{abstract}

Keywords: freight train; energy-efficient operation; maximum principle; periodic braking; numerical algorithm

\section{Introduction}

Freight trains play a major role in cargo transport all over the world. Due to the rising energy consumption, more attention has been paid to freight trains. Taking China as an example, the hauling energy consumption of locomotives accounts for $70 \%-75 \%$ of the total energy consumption of the railways [1]. Thus, due to economic and environmental concerns it's very necessary to reduce the energy consumption of railways.

As we all know, the terrain in China is high in the west and descends toward the east coast. Thus, some rail lines consist of track sections where the train speed still increases even if full (maximum) electrical braking applied, i.e., long-distance steep downhill (LDSD) sections. The train driver must deal with the energy-efficient operation problem of the freight train in LDSD sections. Various methods have been adopted to reduce the energy consumption of freight trains, such as optimizing the timetable, changing the appearance of the locomotive to lower its aerodynamic resistance, installing regenerative brakes to feed the energy back to the traction network [2]. Additionally, a good driving strategy is an economic method with great potential for reducing energy consumption, which is an interesting issue. 


\subsection{Previous Work for the Train Energy-Efficient Operation}

Lots of methods have been utilized to solve the train energy-efficient operation problem since the 1960s [3,4]. Reviewing the approaches, we can divide them into two categories: classic methods and intelligent methods. Classic methods refer to the optimization approaches that use precise mathematical models with rigorous derivations. Intelligent methods are the optimization approaches which solve the train energy-efficient operation problem using multiple objectives and constraints.

\subsubsection{Classic Methods}

Maximum Principle (MP) was firstly employed in the train trajectory optimization by Ichikawa [4]. Then the researchers from Scheduling and Control Group (SCG) improved and developed the application of MP to the problem of train trajectory optimization. Based on MP, three optimal controls for short-distance transport trains were presented by Milroy [5]: maximum power, coast and maximum brake. Asnis et al. [6] proposed that the optimal speed profile consists of four optimal controls: maximum power, cruise, coast and maximum brake. Lee et al. [7] noted that "cruise" was the fourth optimal control for long-distance transport trains. Howlett et al. [8] came up with a new local energy minimization principle to calculate the optimal switching points of the long-haul freight train along a single steep downhill or uphill track section. Albrecht et al. $[9,10]$ discussed the existence and uniqueness of the train operation optimal strategy. The relationship between energy consumption and operation time was studied by Howlett [11]. Meanwhile, other researchers also studied the train trajectory optimization based on MP. Khmelnitsky [12], Golovitcher and Iakov [13], and Liu [14] presented the necessary conditions of the train energy-efficient operation based on MP. Numerical algorithms were used to search for the optimal control strategy when the speed limit was considered in the optimal control problem of the train energy-efficient operation. Yang et al. [15] presented the optimal model of train operation with the regenerative braking considered. And the optimal solution from coasting to braking consists of a set of speed trajectories.

Combined with the Karush-Kuhn-Tucker (KKT) condition and derivative approach, the Lagrangian multipliers method was applied in the trajectory optimization of diesel locomotives with discrete controls. For a train along the level track within a given time, Jiaxin $[16,17]$ noted that the energy consumption of the diesel locomotive was minimized if the velocity of the train was kept close to the critical velocity. Then Cheng's theory was improved by Howlett. The critical velocity was identified as the selected holding speed. The Lagrangian multipliers were introduced to search the energy-efficient driving strategies of a long-haul freight train with discrete control inputs. And the optimal strategy of a freight train on the level track is power-coasting-power between two stations $[18,19]$. For a train on the continuously varying gradient, an iteration method was utilized to find optimal solution to the train energy-efficient operation [20].

With the improvement of computer processing speed, dynamic programming (DP) was applied to the train trajectory optimization. A nonlinear train optimal control problem considering the efficiency of the train traction system was solved via Discrete Dynamic Programming (DDP) [21,22]. Miyatake et al. $[23,24]$ used Bellman's DP to deal with optimization of the running profile of metro and electric trains. To overcome the dimension explosion of DP, sequential quadratic programming (SQP) was applied to the trajectory optimization of a railway vehicle $[25,26]$.

The calculus of variation is also used to solve the train's minimal-energy driving strategy problem. There were two levels in the train control model: discrete control, i.e., the optimal controls such as power, coasting and braking; continuous control such as the traction force varying with the train velocity. A hybrid automata was used to describe the hierarchical control model $[2,27]$.

Mixed-integer linear programming (MILP) that was appropriate for the engineering application was utilized in an automatic train operation (ATO) system to obtain the optimal control sets [28]. The gauss pseudospectral method was applied in the single and multiple trains trajectory optimization [28,29], which was a new approach in the train trajectory optimization in recent years. 


\subsubsection{Intelligent Methods}

Recently many intelligent approaches have been applied to the train trajectory optimization problem, which have the advantage of providing rapid solution to the Multi-Input Multi-Output (MIMO) problems with multiple objectives and constraints. For example, Genetic Algorithm (GA) was used to search for the optimal train speed profile [30]. Based on the necessary conditions derived from MP, GA was proposed to search for the proper coasting points to realize the energy-efficient operation of a train [31]. Zhao et al. [32] presented a multiple train trajectory optimization based on GA to make a compromise between energy use and punctuality when delays occurred. Huang et al. [33] proposed the Multi-population Genetic Algorithm (MPGA) to solve the train energy-efficient operation problem by optimizing the train operation for multiple interstations. Meanwhile, fuzzy predictive control (FPC) was applied to the ATO by Feng [34]. Bai et al. [35] employed FPC to optimize the trajectory of freight trains with diesel locomotives. Ant colony optimization (ACO) was also applied to obtain the single train optimal speed profile [30].

\subsection{Main Contribution}

Train trajectory optimization was studied using various approaches in the previous work [2-35]. Meanwhile, references [8-11] studied the optimal controls of the freight train along the single steep downhill track section, which is not effective for the long steep downhill sections. In brief, the trajectory optimization of the train operation considering the LDSD hasn't been discussed yet. In this paper, the abovementioned problem will be worked out.

This paper provides four main contributions to the literature. Firstly, the mathematical model of the train energy-efficient operation problem considering LDSD has been established. Then the analytical solution is derived. Secondly, the pneumatic braking (or mechanical braking) force is separated from the integration braking force (including electrical braking and pneumatic braking) while deriving the necessary conditions for the energy-efficient operation of the train. Analysis of the adjoint variable shows that the periodic braking strategy should be applied on the LDSD section. Thirdly, we transform the time constraint with respect to the auxiliary reservoir air-filled time (see Section 2.3) to the speed constraint, which could be considered the path constraint in the optimal control problem. Fourthly, the optimal control at the terminal position of the LDSD section is verified.

Although we obtain the analytical solution to the train speed and optimal control, it's not enough to calculate the optimization trajectory. Based on the above conclusions, we give the linkage rules of the speed holding section incorporating LDSD. Then an iterative numerical algorithm is proposed to obtain the optimal speed profile. The comparative simulation results show that the proposed algorithm in this paper saves more energy, which can provide the train driver the significant guidance with respect to the energy-efficient operation issue.

\subsection{Main Structure of this Paper}

This paper is organized as follows: Section 2 proposes the motion model of a freight train with its constraints, which is considered as an optimal control problem; Sections 3 and 4 give the way to solve the aforementioned optimal control problem; Section 5 analyzes the influence of the journey time on the energy consumption between stations; in Section 6, the numerical algorithm is proposed to solve the optimal control problem; Section 7 illustrates the algorithm in the case studies; in Section 8, conclusions and future work are given.

\section{Freight Train Model and Problem Formulation}

\subsection{Real Characteristics of a Freight Train}

Electric locomotives such as China railways HXD1 and HXD2 are utilized for freight transport in the Chinese main line railway sector. If the traction force at low speed stage is set too large, the motor in the locomotive will idle, which would cause wheel slippage. The electrical braking force is similar. 
Here, an HXD2 is chosen as the simulation locomotive, which is shown in Figure 1. The HXD2 that is the source power of a freight train is driven on the track by the adhesion traction force. $v_{l o c}$ is the linear velocity of the wheel in the locomotive. $v_{w}$ is the angular velocity of the wheel in the locomotive, and $v_{l o c}=r \cdot v_{w}$, where $r$ is the radius of the wheel. $N$ is the opposite force from the track and $f_{c}(v)$ is the traction force generated by the motor. $f_{r}(v)$ is the rolling friction.

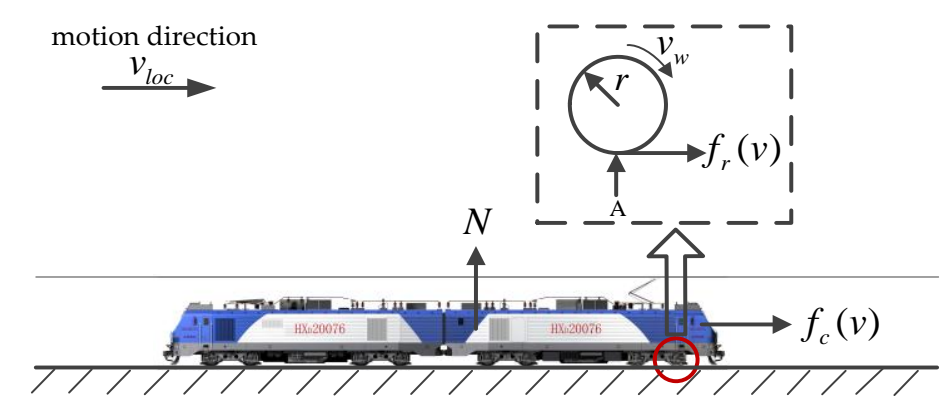

Figure 1. An analytical schematic of a locomotive's three forces.

The rolling friction is caused by the motion of the wheels of the locomotive. Assume that $\mathrm{A}$ is the contact point between the wheel and the track. Since the motion direction of A is opposite to that of the locomotive, then $f_{r}(v)$ occurs to prevent the motion of A as follows:

$$
f_{r}(v)=\mu_{j} N
$$

where $N$ is equal to the weight of the locomotive, $\mu_{j}$ is the rolling friction coefficient of the wheel. It is necessary to point out that $\mu_{j}$ is from a number of field tests [36], i.e.,

$$
\mu_{j}=0.38 \frac{10+0.11 v}{10+0.2 v}
$$

where $v$ is equal to $v_{l o c}$. When a locomotive is hauling, $\mu_{j}$ is the rolling friction coefficient.

In Figure $2 \mathrm{a}, f_{\mathcal{c}}(v)$ is the traction force of the locomotive (blue solid line) and $f_{r}(v)$ is the adhesion traction force of the locomotive (red dash line), which is divided into two parts. The first part (the traction force) that varies linearly with the speed $v_{t}<65 \mathrm{~km} / \mathrm{h}$ is called the "constant torque zone", while the second part (the traction force) that is inversely proportional to the speed $v_{t} \geq 65 \mathrm{~km} / \mathrm{h}$ is called the "constant power zone". Thus, the adhesion traction force varies nonlinearly with the speed. During the low speed stage, the real traction force of a locomotive is less than $f_{c}(v)$ owing to the restriction of the adhesion force. At the high speed stage, the real traction force of a locomotive is equal to $f_{c}(v)$. In short, it can be expressed as:

$$
f_{t 0}(v)=\min \left\{f_{c}(v), f_{r}(v)\right\}
$$

where $f_{t 0}(v)$ is the real traction force of a freight train.

Similar to Equation (2), when a locomotive is using electrical braking, the rolling friction coefficient $\mu_{z}$ is as follows [36]:

$$
\mu_{z}=0.0624+\frac{45.6}{260+v}
$$

where $v$ is equal to $v_{l o c}$. The rolling friction coefficient is:

$$
f_{r z}(v)=\mu_{z} N
$$

In Figure $2 \mathrm{~b}$, the braking force of the HXD2 locomotive is defined as $f_{e b}(v)$, i.e., regenerative braking force (yellow solid line). When the electrical braking is applied, the motor in the locomotive 
will generate electricity. As a result, the electricity is fed back to the traction network so that other trains can utilize it. There are two stages in the electrical braking characteristics. The first changes linearly with the speed $v_{b}<70.6 \mathrm{~km} / \mathrm{h}$, while the latter changes nonlinearly with the speed $v_{b} \geq 70.6 \mathrm{~km} / \mathrm{h}$, i.e., inversely proportional to the speed. $f_{r z}(v)$ that is the adhesion braking force of the locomotive (red dash line) changes nonlinearly with the velocity of the locomotive determined by (5). Thus, the real braking force of the locomotive can be obtained:

$$
f_{d 0}(v)=\min \left\{f_{e b}(v), f_{r z}(v)\right\}
$$

where $f_{d 0}(v)$ is the real regenerative braking force.

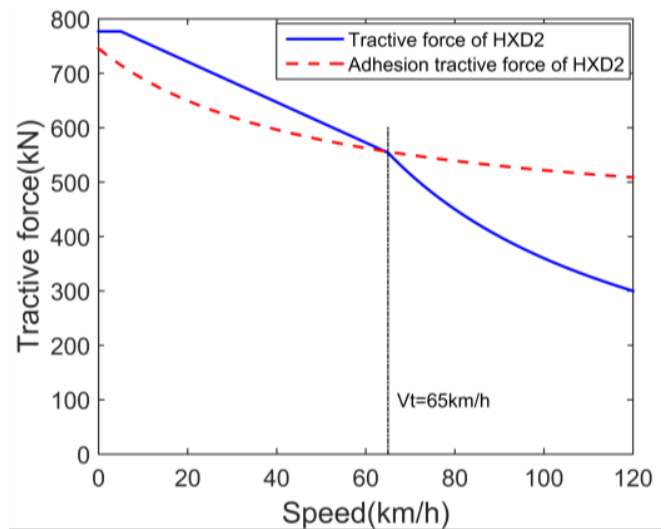

(a)

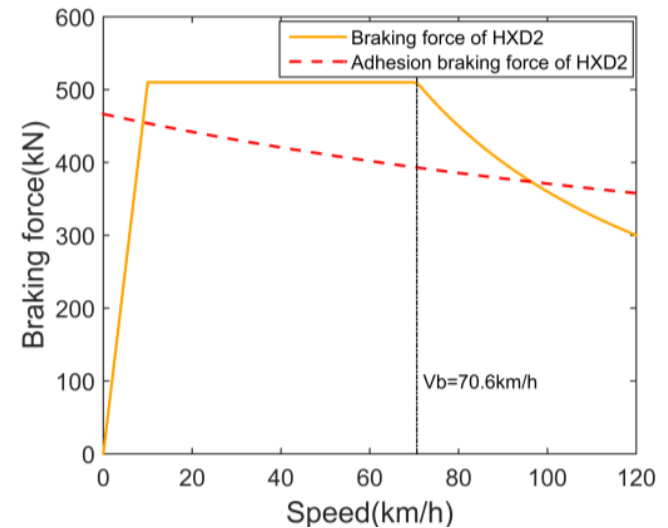

(b)

Figure 2. Traction/Braking force and adhesion force of a locomotive (HXD2): (a) traction force and adhesion force; (b) braking force and adhesion force.

For a freight train, there are two types of braking force. One is the regenerative braking force. The other is mechanical braking force, which is illustrated in [37]. The mechanical braking force is presented as follows (see Figure 3).

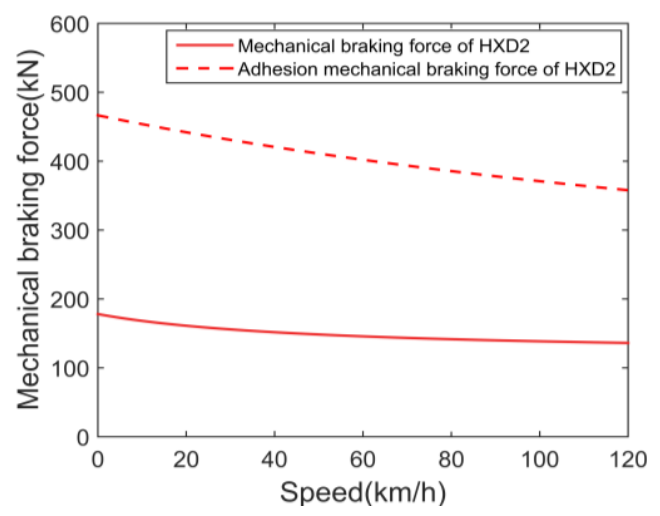

(a)

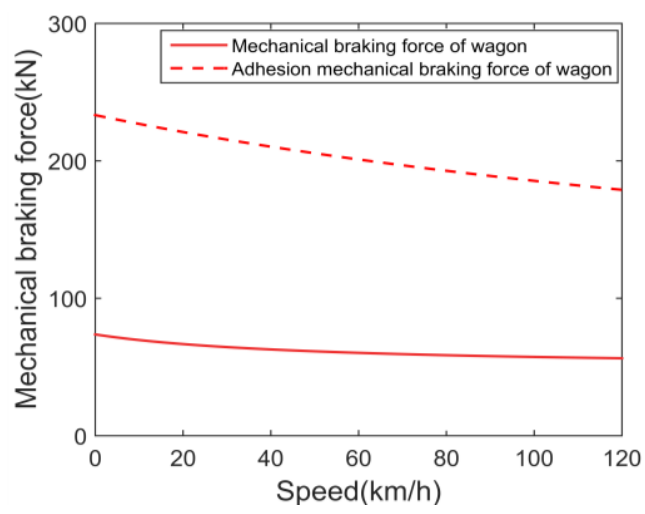

(b)

Figure 3. Mechanical braking force and adhesion force of a freight train: (a) locomotive; and (b) wagon.

In Figure 3, the adhesion force is greater than the mechanical braking force. For a freight train along the track, the mechanical braking force isn't influenced by the adhesion force. The real mechanical braking force can be described [36] using the following expression:

$$
f_{m 0}(v)=\beta_{c} K_{h} \varphi_{h}
$$


where $\beta_{c}$ is the service braking coefficient (equal to 0.17 in this paper). $K_{h}$ is the force of the brake shoe. For a locomotive, it's $580 \mathrm{kN}$. For a fully loaded wagon, it's $195 \mathrm{kN}$. $\varphi_{h}$ is the friction coefficient, which varies nonlinearly with the speed of the train. For a locomotive [36], we have:

$$
\varphi_{h}=0.307 \frac{2 v+150}{3 v+150}
$$

For a fully loaded wagon [36], we have:

$$
\varphi_{h}=0.378 \frac{2 v+150}{3 v+150}
$$

\subsection{Train Model}

The freight train model is proposed in this section. Every wagon in the freight train can possibly run on a section of track with a different gradient. For an example, the front wagon may run on an uphill section while the middle one is on a downhill section. Therefore, a multi-particle model of a freight train is more appropriate in this paper instead of a single-particle one. The train marshalling with one locomotive and $n-1$ wagons is shown in Figure 4.

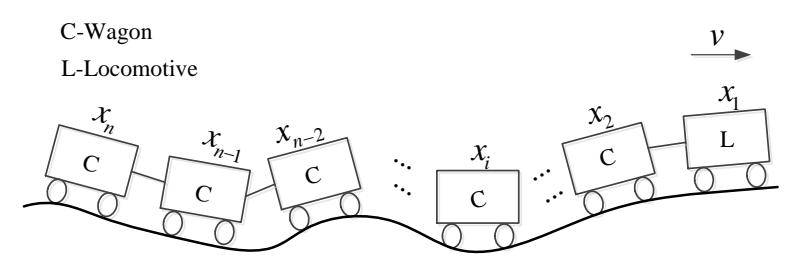

Figure 4. Multi-particle model of a freight train.

Due to the Figure 4 and considering the position of the train $(x)$ as an independent variable, the dynamic equations are as follows:

$$
\begin{gathered}
\frac{d v(x)}{d x}=\frac{\mu_{\mathrm{t}} f_{\mathrm{t}}(v(x))-\mu_{d} f_{d}(v(x))-\mu_{m} f_{m}(v(x))-w_{0}(v(x))-g(x)}{v(x)} \\
\frac{d t(x)}{d x}=\frac{1}{v(x)}
\end{gathered}
$$

where $\mathrm{x}$ is the position of the train, and $x=x_{1}, v(x)$ is the velocity of the train at $\mathrm{x}$, and $t(x)$ is the journey time of the train at $\mathrm{x}$. The control variables: $\mu_{\mathrm{t}} \in[0,1], \mu_{d} \in[0,1], \mu_{m} \in\{0,1\}$, and $\mu_{t} \cdot \mu_{d} \equiv 0, \mu_{t} \cdot \mu_{m} \equiv 0 . f_{t}(v(x))$ and $f_{d}(v(x))$ are the unit traction and electrical braking force of all locomotives in the freight train $(\mathrm{N} / \mathrm{kg})$, respectively. When $M$ is considered as the mass of the freight train, we have $f_{t}(v(x))=f_{t 0}(v) / M$ and $f_{d}(v(x))=f_{d 0}(v) / M f_{m}(v(x))$ is the unit mechanical braking force of the freight train $(\mathrm{N} / \mathrm{t})$ with the relationship $f_{m}(v(x))=\sum_{i=1}^{n} f_{i m 0}(v) / M(\mathrm{~N} / \mathrm{t}), f_{i m 0}(v)(\mathrm{N})$ is the real mechanical braking force of the ith wagon or locomotive. $w_{0}(v(x))(\mathrm{N} / \mathrm{t})$ that is the train unit basic resistance consists of rolling resistance and aero-dynamic resistance. $g(x)$ is made up of two components: unit gradient resistance $g_{\text {grad }}(x)(\mathrm{N} / \mathrm{t})$ and unit curve resistance $g_{\text {curve }}(x)(\mathrm{N} / \mathrm{t}) . w_{0}(v(x))$, $g(x)$ can be described as:

$$
\begin{gathered}
w_{0}(v(x))=a+b v(x)+c v^{2}(x) \\
g(x)=g_{\text {grad }}(x)+g_{\text {curve }}(x)
\end{gathered}
$$

where $a, b$ and $c$ are positive constant coefficients, and the equivalent unit gradient resistance and curve resistance are $g_{\text {grad }}(x)=\sum_{i=1}^{n}\left[g_{\text {grad }}\left(x_{i}\right) \cdot m_{i}\right] / M$ and $g_{\text {curve }}(x)=\sum_{i=1}^{n}\left[g_{\text {curve }}\left(x_{i}\right) \cdot m_{i}\right] / M, m_{i}$ is the mass of ith wagon or locomotive. For simplicity, there is $v=v(x)$. 
Remark 1. The control variable of pneumatic braking is binary, which is different from the continuous control variable of traction and electrical braking. Theoretically, the control variable of pneumatic braking varies continuously with the pressure reduction of the train brake pipe. However, the pressure reduction is discretized based on the operation rules of the freight train in China. The train driver is required to apply the discrete pressure reduction to the freight train. Hence, while modelling the freight train, the discrete pressure reduction is taken into account. And the pneumatic braking force with minimum pressure reduction could make the train speed decrease. Then the binary control variable is introduced- $\mu_{m} \in\{0,1\}$. If $\mu_{m}=0$, the pneumatic braking is not applied. If $\mu_{m}=1$, the pneumatic braking is applied.

\subsection{Problem Formulation}

Let 0 and $X$ be the initial and terminal positions of the freight train. The velocity of the freight train should be 0 at the origin and destination station:

$$
v(0)=0 \quad v(X)=0
$$

Assume the journey time from 0 to $X$ is $T$. The constraint is:

$$
t(0)=0 \quad t(X)=T
$$

Exceeding the line speed limit is not allowed for safety concerns:

$$
v(x) \leq V(x)
$$

where $V(x)$ is the static speed limit at position $x$.

The pressure in the freight train brake pipe increases while the mechanical braking is released [37]. It takes time to recharge the brake pipe to the target pressure $(600 \mathrm{kPa})$. The time is called the auxiliary reservoir air-filled time. To ensure enough braking force is available in the next braking period, the air-filled time should not be greater than the speed rising time [38]:

$$
t_{z} \geq T_{\mathcal{C}}
$$

where $t_{z}$ and $T_{c}$ are the speed rising time and the auxiliary reservoir air-filled time, respectively.

Considering the LDSD section, the energy-efficient operation problem of a freight train that is to find an optimal train trajectory minimizes the energy consumption. Due to the principle of the optimal control, a cost function is defined as follows:

$$
\min J=\int_{0}^{X}\left(\frac{\mu_{t} f_{t}(v)}{\eta_{t}}-\alpha \eta_{d} \mu_{d} f_{d}(v)\right) d x
$$

where $\eta_{t}$ is the energy efficiency ratio of the traction system, $\eta_{d}$ is the energy efficiency ratio of the electrical braking system, $\alpha$ is the regeneration efficiency representing the feedback energy percentage of the electrical braking.

Remark 2. The aim of this paper is to minimize the energy consumption of the traction system (including power and regenerative braking). Our aim is not the same as minimizing total energy consumption of the traction power supply system because the energy efficiency ratios vary with the train speed and the loads, i.e., power and electrical braking. In practice, we could try to turn off some motors in the locomotive at the cruising phase to make the traction system run near the peak efficiency at high load. Then the energy efficiency ratios could be approximatively considered as a constant. 
Remark 3. The regeneration efficiency is introduced for two purposes while modelling the freight train, which is illustrated below:

(1) In most electric trains, the regenerative braking energy can be fed back to the traction power supply system if other trains along the railway line could utilize it. Currently, freight trains are not equipped with on-board energy storage devices. Hence, the regeneration efficiency is decided by the availability of other trains. In the realistic traction power supply system, the regeneration efficiency is a variable. The railway operation scheme (including the timetable and number of freight trains that are running along the railway line) should be investigated to obtain the accurate regeneration efficiency. Based on the operation data in months or years, we could calculate the regeneration efficiency. In China, the railway electrification system is $2 \times 25 \mathrm{kV}$ alternating current $(A C)$ single phase with a frequency of $50 \mathrm{~Hz}$. Two phases of the three-phase AC power from the public power grid are used to supply for the electric trains. The phases at different feeders of the substations will be switched between $A-C, C-B, B-A$, to maintain the overall balance of the public power grid (see Figure 5). Since the double-track railway including up rail and down rail is very common in China, the traction network of up rail and down rail connects in parallel. We can optimize the timetable to make the up train and down train run on the same LDSD section. For example, for a fully loaded train that is running on the up rail of the LDSD section, if electrical braking is applied, the electricity generated by the up train will be fed back to the traction network. At the same time, if the down train with empty wagons is going uphill with power on the LDSD section or the fully loaded up train is powering on the normal gradient (see Figure 5), $\alpha$ of the energy generated by up train will be absorbed by the down train.

(2) In this paper, our aim is to calculate the optimal speed trajectory considering the LDSD section. Our work in this paper is the operation optimization for a single train. The regeneration efficiency is introduced into the optimal control problem to evaluate the ratio of coasting and regenerative braking. To simplify the optimal control problem in this paper, the regenerative efficiency is considered as a constant.

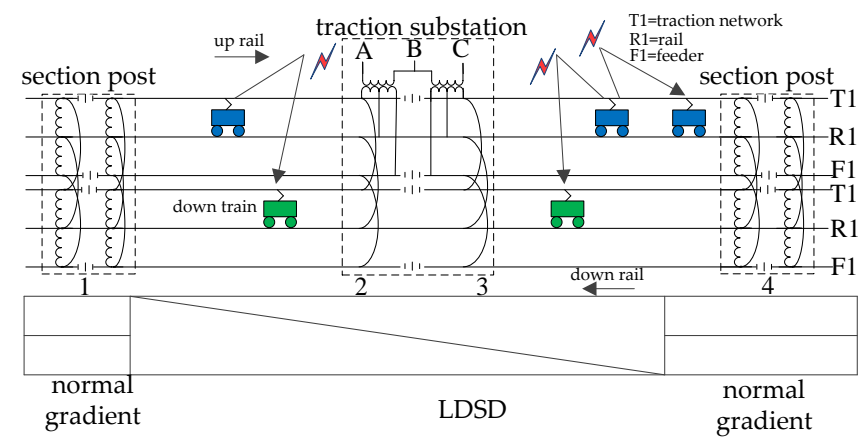

Figure 5. Regenerative energy flow in the traction power supply system.

The problem can be formulated as follows:

Find a series of optimal controls and switching locations to make the cost function (18) minimized. Meanwhile, the dynamic constraints (10) and (11), the path constraints (16), (17), the boundary constraints (14) and (15), must be satisfied.

\section{Solution}

Section 2 gives the formula of the train energy-efficient operation problem. This section discusses the analytical solution to the train trajectory optimization.

\subsection{Necessary Condition of the Train Energy-Efficient Operation}

Combining (10), (11) and (18), the Hamiltonian is as follows: 


$$
H=-\frac{\mu_{t} f_{t}(v)}{\eta_{t}}+\alpha \eta_{d} \mu_{d} f_{d}(v)+\frac{\lambda_{1}}{v}+\frac{\lambda_{2}}{v} \cdot\left[\mu_{t} f_{t}(v)-\mu_{d} f_{d}(v)-\mu_{m} f_{m}(v)-w_{0}(v)-g(x)\right]
$$

where $\lambda_{1}$ and $\lambda_{2}$ are the Lagrange Multipliers.

To simplify (19), a new adjoint variable is introduced:

$$
\theta=\eta_{t} \lambda_{2} / v
$$

which converts Equation (19) as follows:

$$
H=\frac{(\theta-1)}{\eta_{t}} \mu_{t} f_{t}(v)+\left(\alpha \eta_{d}-\frac{\theta}{\eta_{t}}\right) \mu_{d} f_{d}(v)-\frac{\theta}{\eta_{t}} \cdot\left[\mu_{m} f_{m}(v)+w_{0}(v)+g(x)\right]+\frac{\lambda_{1}}{v}
$$

To maximize (21), a set of six optimal controls is presented:

- Full power (FP): $\mu_{t}=1, \mu_{d}=\mu_{m}=0$ when $\theta>1$.

- Partial power (PP): $\mu_{t} \in[0,1], \mu_{d}=\mu_{m}=0$ when $\theta=1$.

- Coasting (C): $\mu_{t}=\mu_{d}=\mu_{m}=0$ when $\alpha \eta_{d} \eta_{t}<\theta<1$.

- Partial electrical braking (PEB): $\mu_{t}=0, \mu_{d} \in[0,1], \mu_{m}=0$ when $\theta=\alpha \eta_{d} \eta_{t}$.

- Full electrical braking (FEB): $\mu_{t}=0, \mu_{d}=1, \mu_{m}=0$ when $0<\theta<\alpha \eta_{d} \eta_{t}$.

- Full braking (FB): $\mu_{t}=0, \mu_{d}=\mu_{m}=1$ when $\theta \leq 0$.

\subsection{Transformation of the Time Constraint}

In this subsection, a theorem about the air-fill time is given. Before that, the necessary definitions and assumption are given.

Definition: Let $v^{\prime}(x)$ be the instantaneous speed from A to B. Let the position of A and B be $x_{A}$ and $x_{B}$. Let the position of $C$ and $\mathrm{D}$ be $x_{C}$ and $x_{D}$. $T_{C}$ is equal to the integration from $x_{A}$ to $x_{B}$ :

$$
T_{\mathcal{C}}=\int_{x_{A}}^{x_{B}} \frac{1}{v^{\prime}(x)} d x
$$

where $v^{\prime}\left(x_{A}\right)=\varphi\left(x_{A}\right)$ and $v^{\prime}\left(x_{B}\right)=V(x)$.

Assumption: suppose $v(x) \leq \varphi(x)$ does not hold, then, there is $v(x)>\varphi(x)$ to assure the existing of the optimal solution.

The Lipschitz continuity should be satisfied:

$$
\left\|F\left(v_{1}, x\right)-F\left(v_{2}, x\right)\right\| \leq L\left\|v_{1}-v_{2}\right\|
$$

where $L \geq 0$.

Theorem 1. Let $\varphi(x)$ be the optimal release speed while the mechanical braking is removed. Let $v(x)$ be the velocity of the train speed. $\varphi$ and $v$ is the function of the train position $x$. If and only if $v(x) \leq \varphi(x)$, the optimal solution to the problem exists. And $T_{c}$ equals to the time from $A$ to $B$ (see Figure 6) by applying full electrical braking (FEB).

Proof of Theorem 1. Proof by contradiction is presented as follows:

Depending on the assumption, $\exists \varphi^{\prime}(x)$ and $\varphi^{\prime}(x)>\varphi(x)$, i.e., $v\left(x_{A}\right)=\varphi^{\prime}\left(x_{A}\right) . \varphi(x)$ and $\varphi^{\prime}(x)$ are constants. To make the time from $v\left(x_{A}\right)$ to $v\left(x_{D}\right)$ equal to $T_{\mathcal{C}}$, there must be a point satisfying:

$$
v\left(x_{C}\right)=v^{\prime}\left(x_{C}\right)
$$


In Figure 6, the instantaneous speed $v(x)$ is always greater than $v^{\prime}(x)$ from $x_{A}$ to $x_{C}$ :

$$
v(x)>v^{\prime}(x)
$$

If $x>x_{C}$, there is:

$$
v(x)<v^{\prime}(x)
$$

which converts Equation (10) into the following expression:

$$
F(v, x)=\frac{d v(x)}{d x}=\frac{-f_{d}(v(x))-w_{0}(v(x))-g(x)}{v(x)}
$$

where $v\left(x_{C}\right)$ and $x_{C}$ are the initial states of the differential Equation (27), i.e., $v=v\left(x_{C}\right), x=x_{C}$. $\forall v_{1}, v_{2}>v\left(x_{C}\right), \forall x>x_{C}, \exists \delta>0$, given the initial condition: Equation (26) and $v=v\left(x_{C}\right)$, only one solution exists at $\left[x_{C}, x_{C}+\delta\right]$.

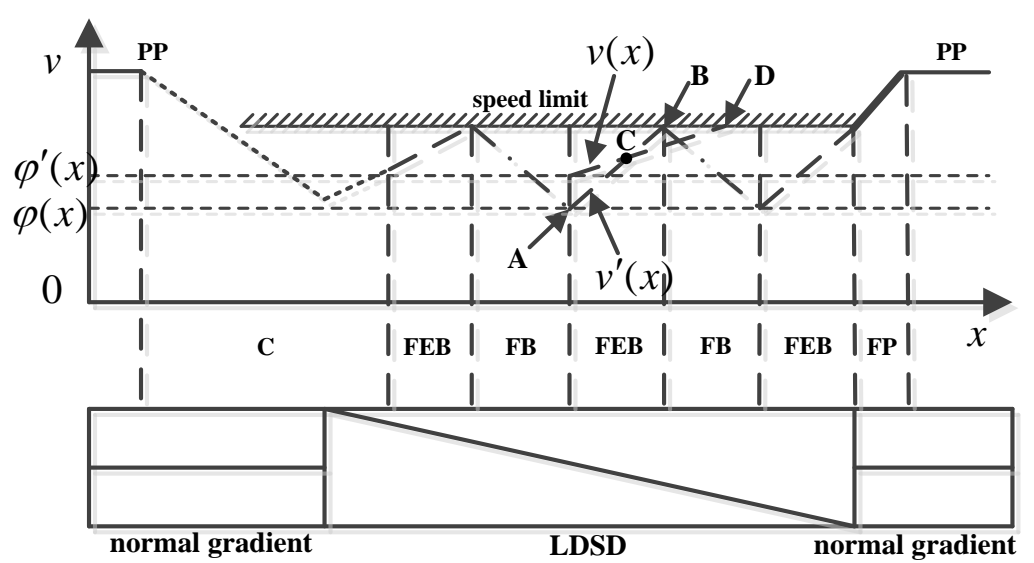

Figure 6. Full electrical braking analysis of the freight train on the long-distance steep downhill (LDSD) section.

However, Equation (26) implies that there are two solutions when $x>x_{C}$. This contradicts the Lipschitz continuity (existence and uniqueness theorem of the differential equation). Then we have:

If $x>x_{C}$, there is:

$$
v(x)=v^{\prime}(x)
$$

which implies $v(x) \geq v^{\prime}(x)$. Meanwhile:

$$
t_{z}=\int_{x_{A}}^{x_{D}} \frac{1}{v(x)} d x \geq \int_{x_{A}}^{x_{B}} \frac{1}{v^{\prime}(x)} d x=T_{C}
$$

Obviously, Equation (29) does not hold if $v(x) \geq v^{\prime}(x)$ and the assumption does not hold. If and only if $v(x) \leq \varphi(x)$, Theorem 1 holds. Finally, the time constraint is transformed to the speed constraint.

\subsection{Solution to the Optimal Control Problem with the Speed Constraint}

The necessary condition of the freight train energy-efficient operation is given in Section 3.1. Further study is proposed to solve the problem with the speed constraint. According to Equation (15) and Theorem 1, two complementary slackness factors $(M(x)$ and $T(x))$ are introduced $[14,38]$ :

$$
\begin{gathered}
{[v(x)-V(x)] d M(x) / d x=0} \\
{[v(x)-\varphi(x)] d T(x) / d x=0}
\end{gathered}
$$


where $v(x)<V(x), d M(x) / d x=0 ; v(x)=V(x), d M(x) / d x>0 . v(x)<\varphi(x), d T(x) / d x=0$; $v(x)=\varphi(x), d T(x) / d x>0$.

The derivative of the Lagrange Multipliers considering the slackness factors is:

$$
\begin{gathered}
\frac{d \lambda_{1}}{d x}=-\frac{\partial H}{\partial t} \\
\frac{d \lambda_{2}}{d x}=-\frac{\partial H}{\partial v}+\frac{d M(x)}{d x}+\frac{d T(x)}{d x}
\end{gathered}
$$

In (31), the Hamiltonian does not depend on $t$ explicitly, the partial derivative of $H$ is zero:

$$
\frac{d \lambda_{1}}{d x}=-\frac{\partial H}{\partial t}=0
$$

thus, $\lambda_{1}=C$, where $C$ is a constant. Equation (20) introduces an adjoint variable to replace the Lagrange Multiplier $\lambda_{2}$. Differentiating (20) yields the following:

$$
d \lambda_{2}=\frac{1}{\eta_{t}} d(\theta v)
$$

which can be rewritten as:

$$
d \lambda_{2}=\frac{1}{\eta_{t}}(v d \theta+\theta d v)
$$

Combining (19), (32) and (35):

$$
d \theta=\left\{\frac{(1-\theta)}{v} \mu_{t} f^{\prime}{ }_{t}(v)+\frac{\left(\theta-\alpha \eta_{d} \eta_{t}\right)}{v} \mu_{d} f^{\prime}{ }_{d}(v)+\frac{\left[w_{0}^{\prime}(v)+\mu_{m} f^{\prime}{ }_{m}(v)\right] \theta}{v}+\frac{\lambda_{1} \eta_{t}}{v^{3}}\right\} d x+\frac{\eta_{t}}{v} d M(x)+\frac{\eta_{t}}{v} d T(x)
$$

In Section 3.1, only the general relationship between the adjoint variable and the train optimal controls is derived, i.e., the necessary condition. However, it's not enough to calculate the precise speed trajectory of the freight train energy-efficient operation. In this section, Equation (36) of the adjoint variable is obtained to determine the optimal controls for the train operation.

\subsection{Analysis of the Special Optimal Controls}

To make the freight train operation energy-efficient, there are six optional optimal controls. Among them, there are three unique optimal controls, i.e., PP, PEB and FB. We will discuss the special optimal controls to get more details about the train energy-efficient operation.

\subsubsection{Partial Power}

When the freight train runs on the interval with PP, there are the adjoint variable $\theta=1$, and its corresponding optimal control $\mu_{t} \in[0,1], \mu_{d}=\mu_{m}=0$. As a result, there is:

$$
d \theta=\left\{\frac{1}{v} w_{0}^{\prime}(v)+\frac{\lambda_{1} \eta_{t}}{v^{3}}\right\} d x+\frac{\eta_{t}}{v} d M(x)+\frac{\eta_{t}}{v} d T(x)
$$

Since $\theta$ is constant, $d \theta / d x=0$. Let $d M(x)=0$ and $d T(x)=0$, (37) can be expressed as:

$$
\frac{d \theta}{d x}=\frac{1}{v} w_{0}^{\prime}(v)+\frac{\lambda_{1} \eta_{t}}{v^{3}}
$$

Equation (38) is rewritten as:

$$
v^{2} w_{0}^{\prime}(v)+\lambda_{1} \eta_{t}=0
$$


where $\lambda_{1}$ and $\eta_{t}$ are constants. When $\theta=1$, there exists one or more optimal constant velocities. To find those, a speed function is defined as follows:

$$
\zeta(v)=v^{2} w_{0}^{\prime}(v)
$$

Due to Equations (12) and (40) can be rewritten as:

$$
\zeta(v)=v^{2}(b+2 c v)
$$

Obviously, $\zeta(v)$ is monotone increasing for $\zeta^{\prime}(v)>0$. When $\theta=1$, only one optimal constant speed $v_{c}$ that makes Equation (39) hold.

Let the target speed of the train operation be $v_{t}$. When $\theta=1$, there is:

$$
v_{t}=\min \left\{v_{c}, V(x)\right\}
$$

The mathematical description of the normal gradient where a freight train can maintain a constant speed is presented:

$$
\mu_{t} f_{t}\left(v_{t}\right)-g(x)-w_{0}\left(v_{t}\right)=0
$$

when the freight train runs on the interval with partial electrical braking (PEB), we have the adjoint variable $\theta=\alpha \eta_{d} \eta_{t}$, and its corresponding optimal control: $\mu_{t}=0, \mu_{d} \in[0,1], \mu_{m}=0$. Substituting $\theta$ into Equation (36) with $\theta=\alpha \eta_{d} \eta_{t}$ yields the following:

$$
d \theta=\left\{\frac{\alpha \eta_{d} \eta_{t}}{v} w_{0}^{\prime}(v)+\frac{\lambda_{1} \eta_{t}}{v^{3}}\right\} d x+\frac{\eta_{t}}{v} d M(x)+\frac{\eta_{t}}{v} d T(x)
$$

Similarly, there is:

$$
\alpha \eta_{d} v^{2} w_{0}^{\prime}(v)+\lambda_{1}=0
$$

which can be rewritten as:

$$
\alpha \eta_{d} \zeta(v)+\lambda_{1}=0
$$

when a freight train is using partial electrical braking, there is only one optimal constant electrical braking speed for Equation (46) due to the monotone increasing of $\zeta(v)$.

Let the target speed of the train operation be $v_{t d}$. When $\theta=\alpha \eta_{d} \eta_{t}$, there is:

$$
v_{t d}=\min \left\{v_{d}, V(x)\right\}
$$

The mathematical description of other type of the normal gradient where a freight train can maintain a constant speed is presented:

$$
-\mu_{d} f_{d}\left(v_{t d}\right)-g(x)-w_{0}\left(v_{t d}\right)=0
$$

\subsubsection{Full Braking}

When the freight train runs on the interval with full braking (FB), $\theta \leq 0$, and the corresponding optimal control: $\mu_{t}=0, \mu_{d}=1, \mu_{m}=1$. Due to the definition of the LDSD, there is:

$$
-\mu_{d} f_{d}(v)-g(x)-w_{0}(v)>0
$$

where $\mu_{d}=1, v \leq V(x), 0<\theta<\alpha \eta_{d} \eta_{t}$.

When pneumatic braking is applied, there is:

$$
-\mu_{d} f_{d}(v)-\mu_{m} f_{m}(v)-g(x)-w_{0}(v)<0
$$


where $\mu_{d}=1, \mu_{m}=1$.

\subsection{Uniqueness Analysis of the Optimal Control at the Terminal Position of the LDSD Section}

Since the freight train operation on a LDSD section is different from that on a normal gradient, the driving strategies that are utilized on the normal gradient can't be used to get through the LDSD section. It's necessary to analyze the optimal controls for the LDSD section.

In this section, a theorem about the uniqueness of the optimal control on the LDSD section will be proved, which gives out the optimal control at the terminal position of the LDSD.

Theorem 2. Assume that a freight train is at the terminal position of the LDSD (see Figure 6). While $v_{\mathcal{c}}>V(x)$, we obtain that the adjoint variable is $0<\theta<\alpha \eta_{d} \eta_{t}$, and the optimal control is FEB, the velocity of the train is equal to the line speed limit.

Proof of Theorem 2. Proof by contradiction:

Assumption: the optimal control at the terminal position of the LDSD is not FEB. Then the possible optimal controls are FP, PP, PEB, C or FB. The comparison of FEB and other optimal controls is presented in Figure 7. In Figure $7 \mathrm{a}-\mathrm{d}, \varphi(x)$ is the pneumatic braking release speed by applying "FB+FEB".

- $\quad \mathrm{FP}$

If the freight train is accelerated to $V(x)$ with FP (see Figure 7a), the air-filled time holds on via Theorem 1 . Then, there is $v(x) \leq \varphi_{a}(x)$, where $\varphi_{a}(x)$ is the pneumatic braking release speed by applying "FB+FP". Since the acceleration of FP is greater than that of FEB, $\varphi_{a}(x)$ is far less than $\varphi(x)$ so that there is sufficient air in the auxiliary reservoir for the next pneumatic braking. Therefore, it's very possible that $\varphi_{a}(x)$ is less than $30 \mathrm{~km} / \mathrm{h}$ [39], which will cause large longitudinal impact on the wagon couplers. Meanwhile, the freight train is running on the energy-efficient slope, i.e., LDSD section. It does not necessarily consume energy from the traction network. In summary, FP is not the optimal control at the terminal position of the LDSD.

- $\quad$ PP

Equation (38) can be rewritten as:

$$
\frac{d \theta}{d x}=\frac{\zeta(v)+\lambda_{1} \eta_{t}}{v^{3}}
$$

In Figure $7 \mathrm{~b}, \varphi_{b}(x)$ is assumed as the holding speed or pneumatic braking release speed with the relationship $\varphi_{b}(x)<v_{\mathcal{c}}$. If the trip time is given, Section 3.4.1 implies that there is only one optimal holding speed $v_{c}$ to make $d \theta / d x=0$. Meanwhile, $\zeta(v)$ is monotone increasing. When $\varphi_{b}(x)<v_{c}$, there is $d \theta / d x<0$, i.e., $\theta$ is monotone decreasing.

For a freight train that is running at $x_{1}$ with PP $\left(v\left(x_{1}\right)=\varphi_{b}\left(x_{1}\right)\right)$, the corresponding adjoint variable $\theta\left(x_{1}\right)=1$. If the train runs from $x_{1}$ to $x_{2}$ with $\mathrm{PP}$, we have $v\left(x_{2}\right)=\varphi_{b}\left(x_{2}\right)$ and $\theta\left(x_{2}\right)=1$. Since $\varphi_{b}(x)$ is the holding speed, then $\varphi_{b}(x)=\varphi_{b}\left(x_{1}\right)=\varphi_{b}\left(x_{2}\right)$. Once $\theta$ is monotone decreasing, then the adjoint variable $\theta\left(x_{2}\right)<1$. Obviously, the optimal control for the freight train at $x_{2}$ is not PP. However, this contradicts with the assumption: the train should be running on the LDSD with PP after pneumatic braking is released. Thus, PP is not the optimal control.

\section{- $\quad$ PEB}

In a similar way, it can be proved that the velocity of the train is not maintained if PEB is applied on the LDSD section, which contradicts with the assumption-the train should be running at constant speed $v_{d}$ based on Section 3.4.2. Then, PEB is excluded. 
- $\mathrm{C}$

According to Equation (18), there is $\min J_{1}=0$ at the coasting phase (see Figure $7 \mathrm{c}$ ). On the other hand, while FEB is applied, the energy consumption is:

$$
\min J_{2}=\int_{0}^{X_{2}}\left(-\alpha \eta_{d} \mu_{d} f_{d}(v)\right) d x
$$

where $X_{2}$ is the running distance by applying FEB.

It is clear that $\min J_{2}<\min J_{1}$. Thus, it's more energy-efficient to apply FEB. Meanwhile, if FEB is applied, the electricity generated from the motor in the locomotive can be fed back to the traction network so that other trains absorb it. If $C$ is applied, the pneumatic braking speed $\varphi_{c}(x)$ that may be very low leads to large longitudinal impact on the couplers. Thus, $\mathrm{C}$ is not the optimal control.

\section{- $\mathrm{FB}$}

Assume that $\varphi_{d}(x)$ is the pneumatic braking release speed by applying "FEB+FB" (see the blue line in Figure $7(\mathrm{~d}))$. We have $\varphi(x)=\varphi_{d}(x)$. Compared with the energy consumption of accelerating the train speed from $v_{c}$ to $V(x)$, more traction energy is consumed if the train speed is accelerated from $v_{c}$ to $\varphi_{d}(x)$ (the blue line). Therefore, FB is not the optimal control. In summary, the assumption does not hold. The only optimal control is FEB. Theorem 2 states uniqueness of the optimal control at the terminal position of the LDSD.

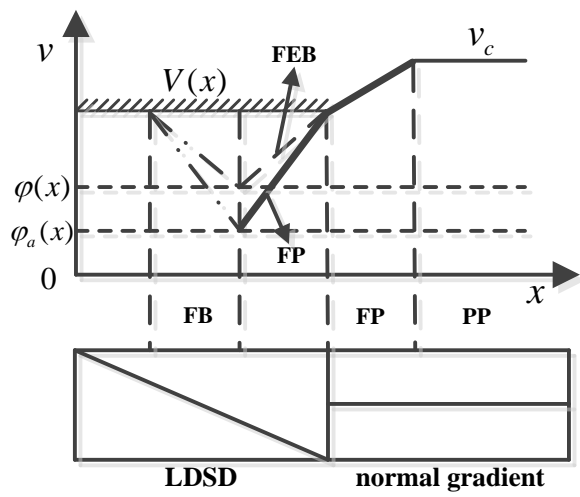

(a)

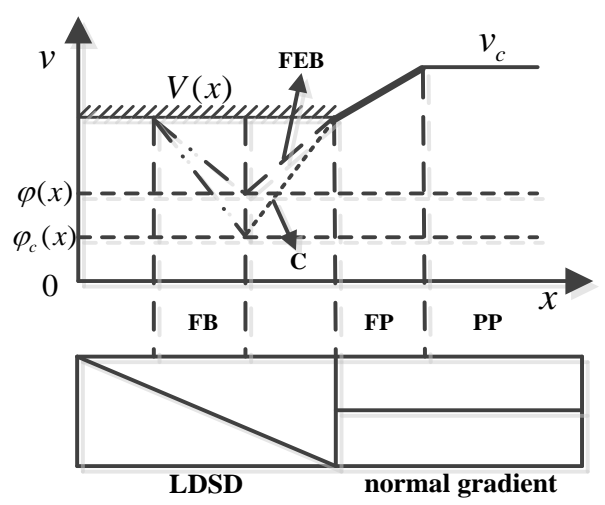

(c)

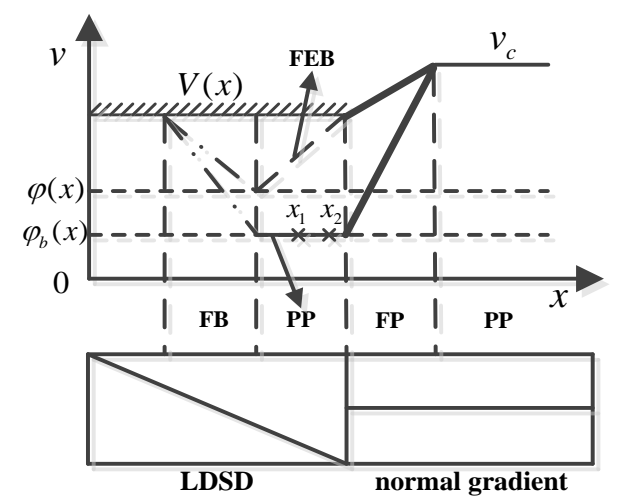

(b)

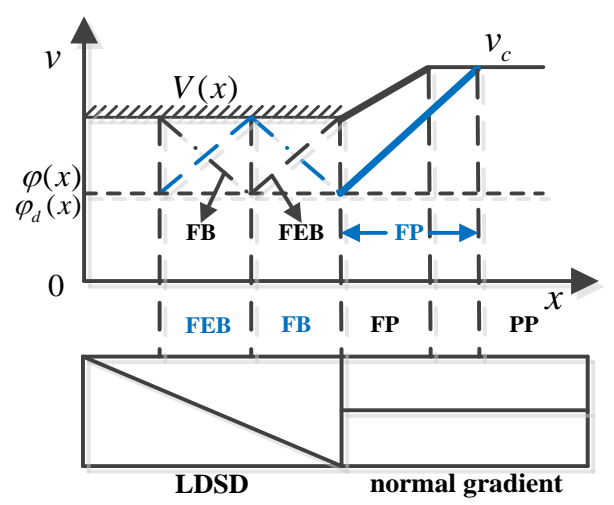

(d)

Figure 7. Comparative analysis of the optimal controls: (a) FEB and FP; (b) FEB and PP; (c) FEB and C; (d) FEB and FB. 


\section{Linkage of the Speed Holding Section}

\subsection{Direction of the Linkage}

Let the speed holding section be the interval with PP or PEB. The corresponding velocities are $v_{c}$ and $v_{d}$. The optimization trajectory from the initial position $(0)$ to the terminal position $(X)$ is divided into three parts. Figure 8 shows the linkage of the three different parts.

As Theorem 1 and Theorem 2 should be satisfied in the optimization trajectory calculation, a backward integration (profile 1) is adopted to calculate the speed profile in the LDSD section. And a forward integration is adopted to calculate the speed profile in the normal gradient section. The two profiles meet at the switching position $x_{1}$ (see Figure 8), while the adjoint variables of the two speed profiles also meet at $x_{1}$.

Meanwhile, another backward integration (profile 2) is adopted to calculate the speed profile from the terminal position $(X)$ to the end position of the LDSD section. Backward profile 1 and backward profile 2 meet at the switching position $x_{2}$. And the adjoint variables of the two speed profiles also meet at $x_{2}$.

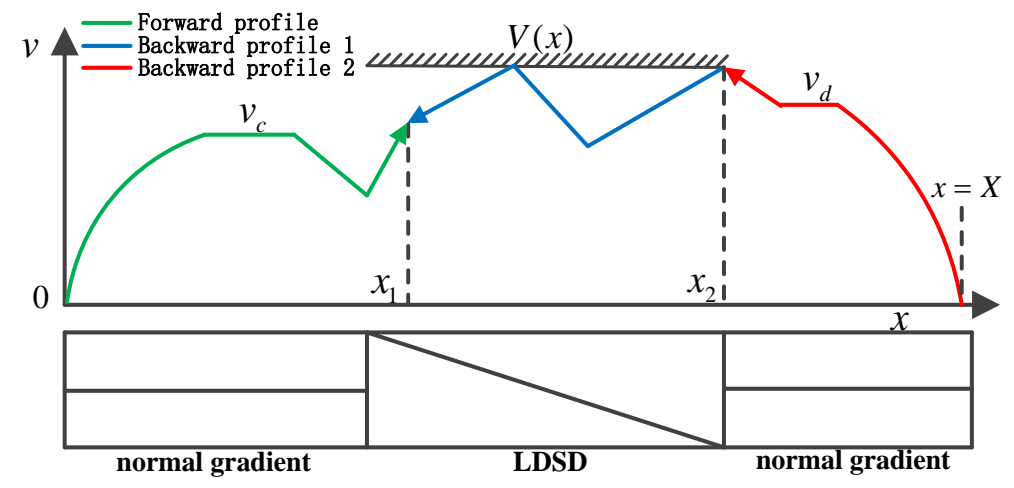

Figure 8. Forward and backward speed profile.

\subsection{Sufficient Conditions of State Variable Inequality Constraint}

Seierstad and Sydsaeter [40] proposed a sufficient theorem to deal with the state variable inequality constraint (Equations (16) and (17)). Since $\theta$ is the function of the train position $x$, a piecewise continuous function $\theta(x)=\left[\theta_{1}(x), \theta_{2}(x), \cdots, \theta_{n}(x)\right]$ is assumed to be existed on the span $[0, X]$ which has a piecewise continuous derivative $-d \theta / d x$ (see Equation (36)) except for a finite number of discontinuous points. At the points of discontinuity $\tau_{1}, \tau_{2} \cdots \tau_{k}$ of $\theta(x), 0<\tau_{1}<\tau_{2}<\cdots<\tau_{k} \leq X$, the following jump condition is satisfied:

$$
\theta_{j}\left(\tau_{i}^{+}\right)=\theta_{j}\left(\tau_{i}^{-}\right)-\left.b \frac{\partial h(x, v(x))}{\partial v}\right|_{x=\tau_{i}}
$$

where:

- $\tau_{i}^{-}$and $\tau_{i}^{+}$denote the left-hand side and the right-hand side of the train position $\tau_{i}$.

- $j=1,2, \cdots, n$.

- $\quad i=1,2, \cdots, k$.

- $b \geq 0$.

- $h(x, v(x))=V(x)-v(x) \geq 0$ or $h(x, v(x))=\varphi(x)-v(x) \geq 0$.

According to the Equation (30), the jump condition of $\theta$ are as follows:

(1) While $v(x)=V(x), d M(x) / d x>0, \theta$ jumps.

(2) While $v(x)=\varphi(x), d T(x) / d x>0, \theta$ jumps. 


\subsection{Linkage Case}

Two typical cases are used to illustrate the linkage of the speed holding section incorporating the LDSD section, i.e., $v_{c}>V(x)$ and $v_{c}<V(x) . \theta$ is the function of the train position $x$. The relationship of $\theta$ and $x$ is presented.

\subsubsection{Case 1}

(1) In Figure 9, the optimal control of the freight train switches from PP to C before entering the LDSD section. Based on the linkage direction in Section 4.1, the intersection of forward profile (C) and backward profile 1 (FEB) is $x=x_{0}$.

(2) Entering the LDSD section, the speed of the freight train keeps increasing even if FEB is applied. The adjoint variable $(\theta)$ is still decreasing. While $\theta=0^{-}, v(x)=V(x)$. Although the jump condition of $\theta$ is satisfied, the jump will not happen yet due to the optimal controls. At the next moment, $\theta=0^{+}$, FB is applied. We have $v(x)<V(x)$.

(3) Once FB is applied, pneumatic braking is released until $v(x)=\varphi(x)$. Then $d T(x) / d x>0, \theta$ jumps at $\tau_{1}^{-}$. We have $0<\theta\left(\tau_{1}^{+}\right)<\alpha \eta_{d} \eta_{t}$, then FEB is applied. And FEB is kept until $v(x)=V(x)$ and $\theta=0^{-}$. Then FB is applied again.

(4) While the freight train is running at the terminal position of the LDSD section, we have $v\left(\tau_{3}^{-}\right)=V\left(\tau_{3}^{-}\right)$. For $d M(x) / d x>0, \theta$ jumps from $\tau_{3}^{-}$to $\tau_{3}^{+}$. Then FP is applied while the freight train is leaving the LDSD. Finally, the freight train is accelerated to $v_{c}$.

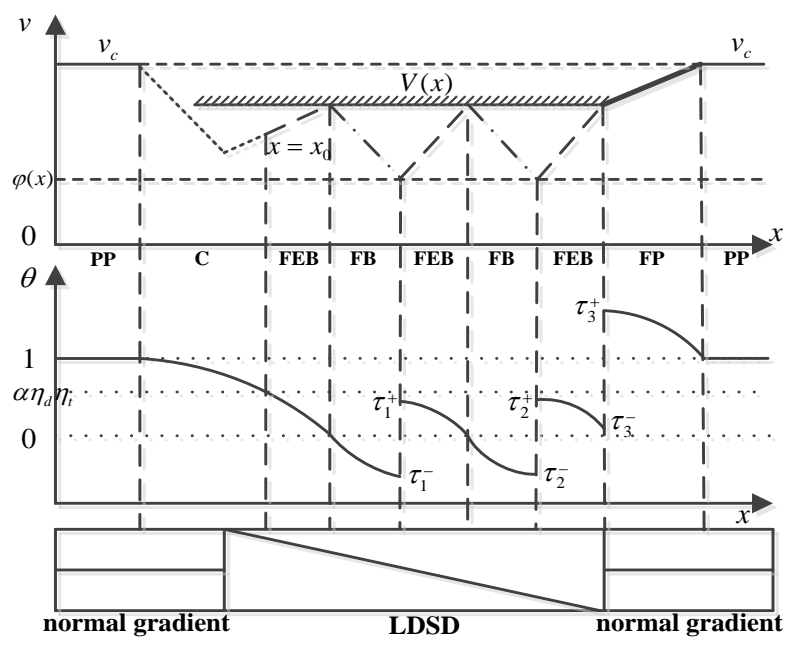

Figure 9. Linkage of the speed holding section considering the LDSD section $\left(v_{c}>V(x)\right)$.

\subsubsection{Case 2}

(1) In Figure 10, the optimal control of the freight train switches from PP to $\mathrm{C}$ before entering the LDSD section. Based on the linkage direction in Section 4.1, the intersection of forward profile (C) and backward profile 1 (FEB) is $x=x_{0}$.

(2) While $\theta=0^{-}, v(x)=V(x)$. At the next moment, $\theta=0^{+}$, FB is applied. We have $v(x)<V(x)$.

(3) Once FB is applied, pneumatic braking is released until $v(x)=\varphi(x)$. Then $d T(x) / d x>0, \theta$ jumps at $\tau_{1}^{-}$. We have $0<\theta\left(\tau_{1}^{+}\right)<\alpha \eta_{d} \eta_{t}$, FEB is applied. And FEB is kept until $v(x)=V(x)$ and $\theta=0^{-}$. Then FB is applied again.

(4) While the freight train is running at the terminal position of the LDSD section, we have $v\left(\tau_{2}^{-}\right)=V\left(\tau_{2}^{-}\right)$. For $d M(x) / d x>0, \theta$ jumps from $\tau_{2}^{-}$to $\tau_{2}^{+}$. Then FP is applied while the freight train is leaving the LDSD. Finally, the freight train will coast to $v_{d}$. And the optimal control between PEB section and PP section is C. 


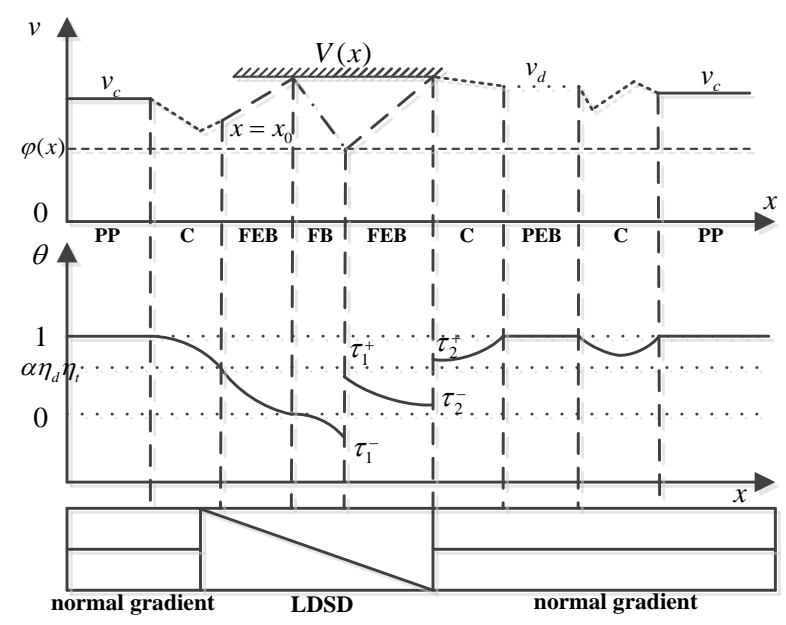

Figure 10. Linkage of the speed holding section considering the LDSD section $\left(v_{c}<V(x)\right)$.

\section{Relationship between Journey Time and Energy Consumption}

In the previous articles $[2,11,41]$, the conflicted relationship between the journey time and energy consumption was studied. From the perspective of traction energy consumption, we draw a conclusion: the total energy consumed $(E)$ is a rigorously monotone decreasing function of the given journey time $\left(T_{0}\right)$. The relationship is presented in the following mathematical equation:

$$
E=\psi\left(T_{0}\right)
$$

where $\psi^{\prime}\left(T_{0}\right)<0$.

The relationship can be illustrated in Figure 11 .

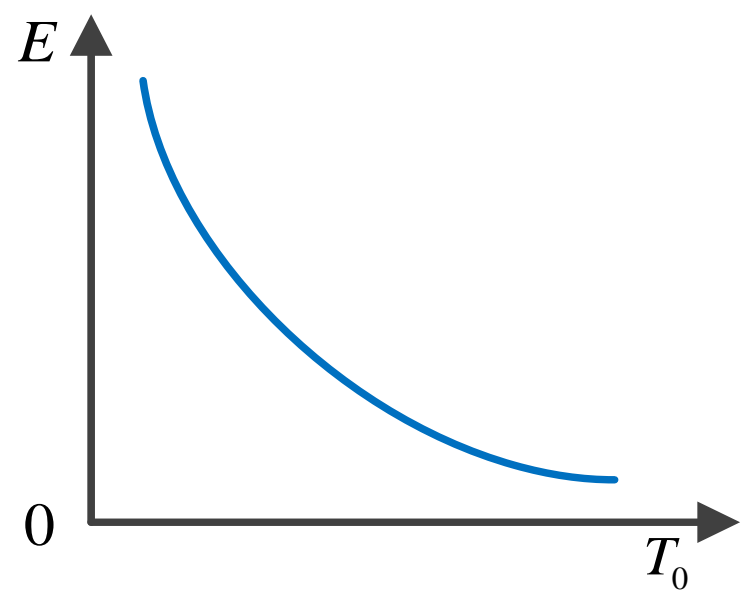

Figure 11. Energy consumption and journey time.

The conclusion will be adopted to analyze the relationship of the interval time allocation and the freight train's energy consumption. Depending on Theorem 2 and Section 4.3, the optimal driving strategy on the LDSD section should be the periodic braking, i.e., FEB+FB+FEB (see Figure 12), which increases the number of freight trains that pass Interval 2 and this decreases the total energy consumption from 0 to $X$. 


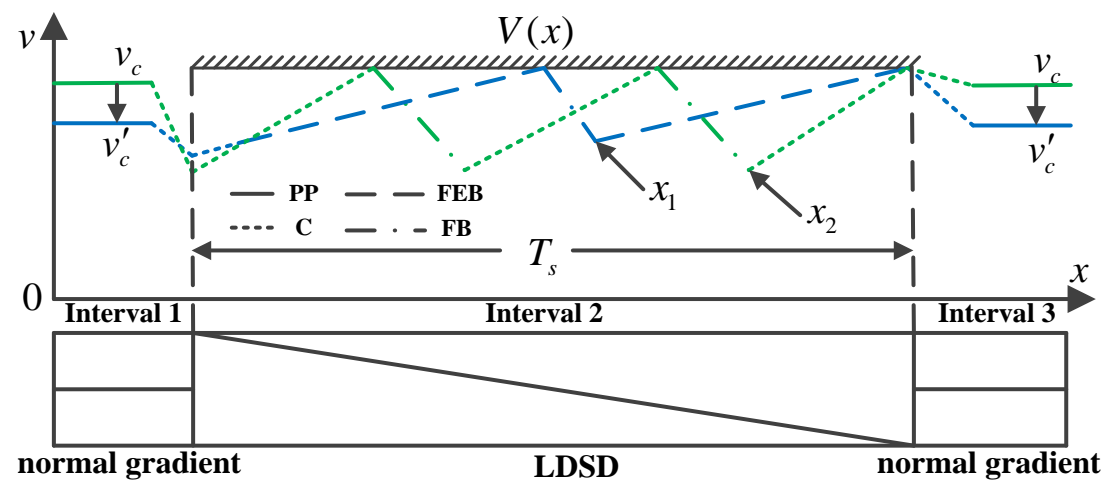

Figure 12. Interval time allocation and energy consumption.

Analysis: in Figure 12, suppose there are two driving strategies on the LDSD section.

(1) Strategy 1: FEB+FB+FEB, the pneumatic braking release speed is equal to $\varphi\left(x_{1}\right)$.

(2) Strategy 2: $\mathrm{C}+\mathrm{FB}+\mathrm{C}$, the pneumatic braking release speed is equal to $\varphi\left(x_{2}\right)$.

The trip from 0 to $X$ is divided into three intervals as follows. $v_{c}$ and $v^{\prime}{ }_{c}$ are the holding speed (PP) of Interval 1 and Interval 3. $T_{s}$ is the journey time of Interval 2. Given the total journey time $T_{s e t}$, the journey time of Interval 1 and Interval 3 is $T_{\text {set }}-T_{s}$.

As $\varphi\left(x_{1}\right)>\varphi\left(x_{2}\right)$, the journey time of Strategy 1 is less than that of Strategy 2 in Interval 2, which means that the average speed of the freight train with Strategy 1 is greater than that with Strategy 2. Obviously, the number of the pneumatic braking instances with Strategy 1 should be less than that with Strategy 2 because the average speed with Strategy 1 is greater. Therefore, the number of freight trains that pass Interval 2 in the fixed time has increased. Meanwhile, if Strategy 1 is applied in Interval 2 , the journey time $T_{s} \downarrow$, then the journey time of the rest intervals $\left(T_{s e t}-T_{s}\right) \uparrow$. And the holding speed drops down from $v_{c}$ to $v^{\prime}$. According to the relationship between the energy consumption and the journey time (see Figure 11), the traction energy consumption of Strategy 1 is less than that of Strategy 2. Meanwhile, Strategy 1 and Strategy 2 will generate electricity on the LDSD section and feed back to the traction network. In Interval 2, the full electrical braking distance of Strategy 1 is greater that of Strategy 2 and the average electrical braking force of Strategy 1 is also greater than that of Strategy 2. Then we could infer that Strategy 1 generates more energy than that of Strategy 2. Hence, considering the energy consumption of Interval 1 Interval 3, we can conclude that Strategy 1 is more energy-efficient.

\section{Numerical Algorithm}

In this paper, the necessary condition of the train energy-efficient operation is derived. The linkage of the speed holding section is given. The constraints (13), (16) and (17) are considered in the optimal control problem. However, to obtain an optimal trajectory of the train operation under the given time, the constraint (15) should be taken into consideration. An iterative algorithm is proposed to calculate the optimal trajectory of the freight train energy-efficient operation incorporating the LDSD section. It's difficult to choose an initial holding speed $\left(v_{\mathcal{c}}\right)$ with PP, in the first iteration, suppose the initial holding speed is:

$$
v_{c}=\frac{X}{T_{\text {set }}}
$$

The detailed procedure of the algorithm is as follows:

(1) Calculate the shortest time $\left(T_{1}\right)$ from 0 to $X$, obtain the flat-out running trajectory.

- If $T_{\text {set }} \geq T_{1}$, then go to step (2). 
- If $T_{\text {set }}<T_{1}$, optimization terminates, the flat-out running trajectory is returned.

(2) Initializing $v_{c}$, then solve $v_{d}$ by combining (39) and (45). Find the speed holding interval with PP and PEB, exclude the speed holding intervals where the freight train can't keep constant speed with FP. If $v_{c}>V(x)$ and $v_{d}>V(x)$, the solution is (42) and (47).

(3) Generate the slope partition table which consists of the initial and terminal position of the speed holding interval. Suppose the set $(U)$ represents the slope partition table. We have $U=\left\{P_{1}, P_{2}, \cdots, P_{i}, \cdots, P_{n}\right\}$, where, $n$ is the number of the speed holding interval.

(4) Linkage of the speed holding intervals.

- Linkage of the adjacent speed holding intervals.

Suppose $i<n$, and $j=i, k=i+1$. Connect $P_{j}$ and $P_{k}$. If the connection of the two speed holding intervals fails, then jump to the linkage of the non-adjacent speed holding intervals.

- $\quad$ Linkage of the non-adjacent speed holding intervals (see Figure 13).

Suppose $j=n-1, k=n$. The last speed holding interval that connects with $P_{j}$ successfully is $P_{m}(1 \leq m<j) . m$ is equal to $n-2$. Connect $P_{m}$ and $P_{k}$. If the connection fails, repeat until the connection succeeds in the domain of $m$, i.e., $m \in[1, j)$.

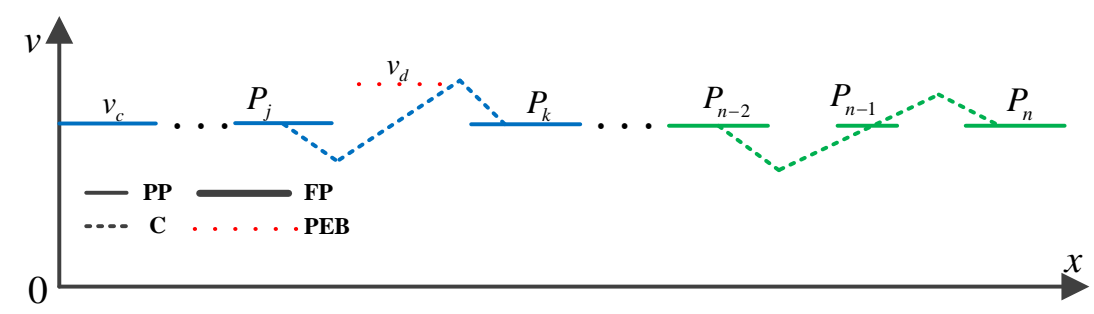

Figure 13. Linkage of the non-adjacent speed holding interval.

Suppose $k<n$ and $j=k-1$, then $k=k+1$, and connect $P_{j}$ and $P_{k}$. If the connection fails, repeat until the connection succeeds in the domain of $j$, i.e., $j \in[1, k-1)$.

(5) The speed profile and optimal controls during the whole trip are recorded. Calculate the train operation time from 0 to $X$. We have $T$.

(6) Evaluate the terminal condition:

$$
\left|T-T_{\text {set }}\right| \leq 5 s
$$

If (56) is satisfied, optimization terminates. Or, if $T>T_{\text {set }}$, increase $v_{c}$; if $T<T_{\text {set }}$, decrease $v_{c}$.

(7) Repeat steps $2-6$ until the terminal condition is satisfied.

(8) Return the optimal trajectory, optimal controls and energy consumption.

(9) Optimization terminates.

\section{Case Study}

This section illustrates the methodology with case studies. The optimization algorithm is implemented on a computer equipped with $2.4 \mathrm{GHz}$ Core i7 processor and 4GB RAM. Matlab 2014b is adopted to simulate the train operation.

The energy-efficient operation of a freight train with one locomotive (HXD2) and 100 fully loaded wagons is studied. The track interval from Yanqing to Chawu is chosen as the case study line, which is part of Daqin Railway Line with the track length $X=69.445 \mathrm{~km}$. The main parameters of the freight train are presented in Table 1 . The speed limits are listed in Table 2. The real traction and braking force are illustrated in Section 2.1. 
Table 1. Parameters of the freight train in the simulation.

\begin{tabular}{ccc}
\hline Parameter & Value & Unit \\
\hline Train marshalling & 1 locomotive +100 wagons & - \\
Locomotive mass & 200 & $\mathrm{t}$ \\
Locomotive length & 38 & $\mathrm{~m}$ \\
Maximum velocity & 120 & $\mathrm{~km} / \mathrm{h}$ \\
Maximum traction force & 777 & $\mathrm{kN}$ \\
Locomotive unit basic resistance & $11.772+0.063765 v+0.002737 v^{2}$ & $\mathrm{~N} / \mathrm{t}$ \\
Energy efficiency ratio of the & 0.9 & - \\
traction system & & \\
Energy efficiency ratio of the & 0.9 & - \\
electrical braking system & 0.9 & - \\
Regenerative coefficient & 100 & $\mathrm{t}$ \\
Mass per one wagon & 12.2 & $\mathrm{~m}$ \\
Length per one wagon & 130 & $\mathrm{~N} / \mathrm{t}$ \\
Wagon unit basic resistance & $9.0252+0.047088 v+0.001226 v^{2}$ & $\mathrm{~s}$ \\
\hline Auxiliary reservoir air-filled time & &
\end{tabular}

Table 2. Line speed limits.

\begin{tabular}{ccc}
\hline Origin Location $\mathbf{( k m )}$ & Terminal Location $\mathbf{( k m )}$ & Value $\mathbf{( k m} / \mathbf{h})$ \\
\hline 0 & 2.9 & 35 \\
2.9 & 67 & 75 \\
67 & 67.720 & 70 \\
67.720 & 70 & 35 \\
\hline
\end{tabular}

\subsection{Train Operation Simulation under Different Journey Time}

The optimization algorithm is implemented based on Section 6. The optimal trajectories under the four groups of the journey time are calculated, which are shown in Figure 14.

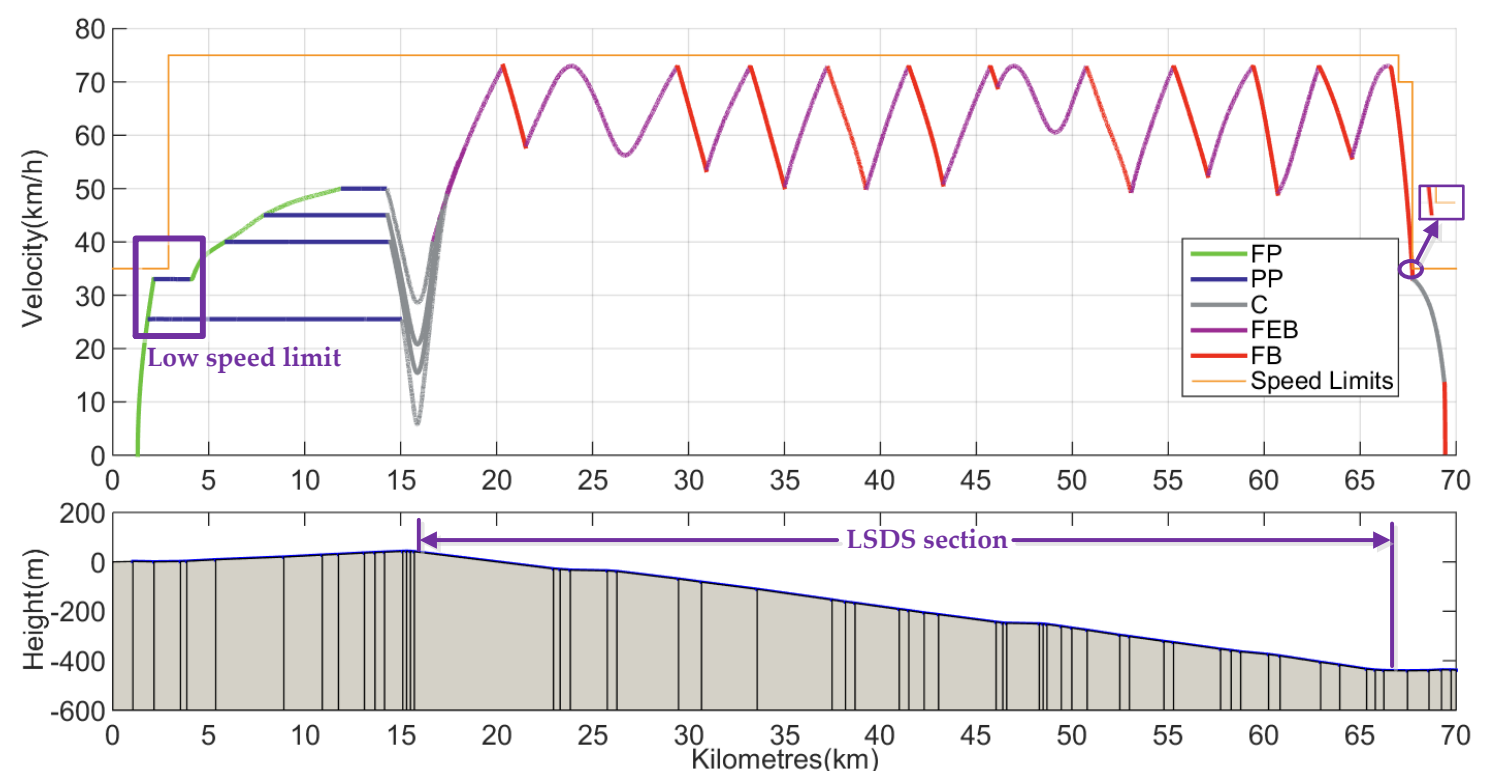

Figure 14. Optimal velocity trajectories and track height.

The track gradient is transformed to the track height for observation convenience. Assume that the track height is $0 \mathrm{~km}$ at the origin location, then the track height of other location is equal to the sum of multiplying the gradient value by the gradient length from $0 \mathrm{~km}$ to current train location. 
Now we obtain four optimal trajectories by using the multi-particle model of the freight train. At the start phase, the freight train is running with FP. The velocity of the freight train must be kept less than the speed limit ( $2 \mathrm{~km} / \mathrm{h}$ below the speed limit). To prevent the rear of the freight train from exceeding the speed limit, the velocity is accelerated until the last wagon passes the low speed limit (see Figure 14). To make the train operation energy-efficient, the optimal control should be switched from PP to $C$ before entering the LDSD section. In the LDSD section, periodic braking is applied and the optimization trajectories are the same under the four groups of given time because the jump condition (see Sections 4.2 and 4.3) should be satisfied. The simulation results of the optimization algorithm are listed in Table 3.

Table 3. Simulation results under different journey time.

\begin{tabular}{ccccc}
\hline Journey Time $(\mathbf{s})$ & $\boldsymbol{v}_{\boldsymbol{c}} \mathbf{( \mathbf { k m } / \mathbf { h } )}$ & Energy Consumption $\mathbf{( k W h )}$ & Regenerative Energy(kWh) & Total Energy Consumption (kWh) \\
\hline 5704.20 & 25.5 & 1701.85 & 5583.39 & -3323.20 \\
4914.49 & 40 & 1784.06 & 5608.62 & -3263.70 \\
4779.73 & 45 & 1821.44 & 5627.82 & -3243.60 \\
4682.57 & 50 & 1873.84 & 5667.86 & -3227.23 \\
\hline
\end{tabular}

The operation energy of different times is listed in Table 3. The total energy consumption is defined as:

$$
J=J_{t}-\alpha \cdot J_{d}
$$

where $J$ is the total energy consumption, $J_{t}$ is the energy consumption of the freight train operation with FP and $\mathrm{PP}, J_{d}$ is the regenerative braking energy of the train operation with electrical braking. In Table 3, the total energy consumption is less than 0, which implies that the train generates more energy than it consumes in the given track interval. Meanwhile, reducing the journey time will cause the energy consumption and total energy consumption to increase. The relationship of journey time and energy consumption/total energy consumption is presented in Figure 15.

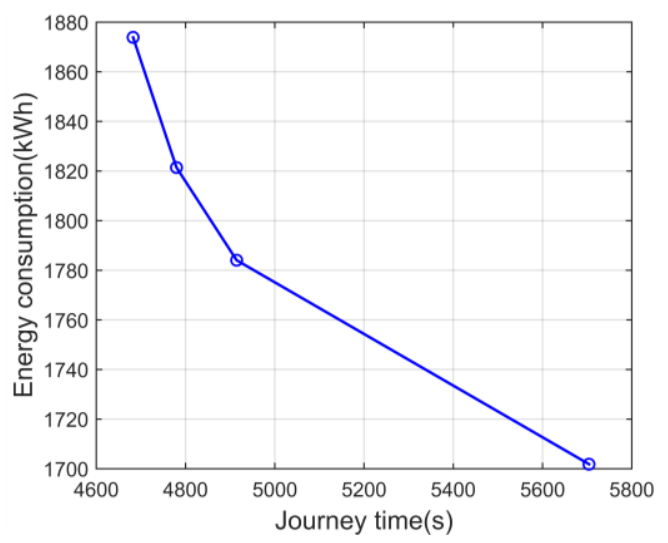

(a)

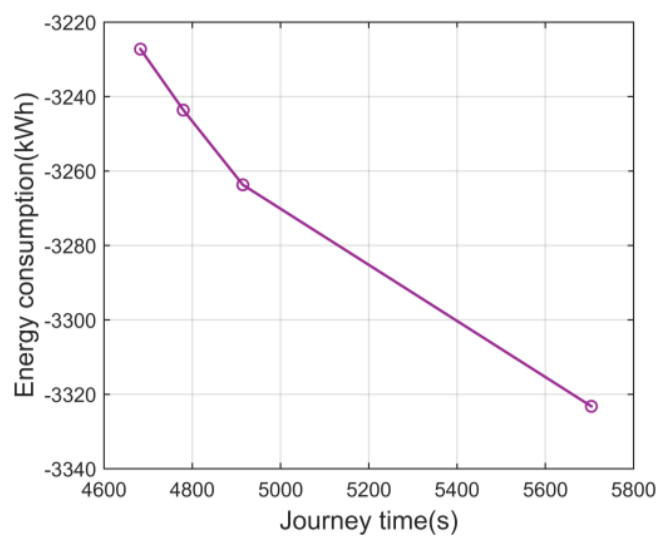

(b)

Figure 15. Relationship between energy consumption/total energy consumption and journey time:

(a) Energy consumption and journey time; (b) total energy consumption and journey time.

In Figure 15a, the traction energy consumption drops down while the trip time of the train increases. Less energy is consumed if the train runs more slowly. It seems the train driver should drive very slowly. However, from the railway system's perspective, reducing the journey time not only allows more freight trains to operate on the railway line, but also creates more economic benefit. Dispatchers of the railway should make a compromise between the energy consumption and the trip time. 
Similarly, the total energy consumption is also monotonically decreasing while the journey time of the train increases even if the regenerative energy consumption is considered. It means that more energy will be fed back to the traction network if the trip time is longer.

\subsection{Comparison between The Proposed Algorithm and Other Methodologies}

In Section 7.1, the optimal trajectories under different journey time are calculated. The simulation result shows that the energy consumption is monotonically decreasing while the holding speed $v_{\mathcal{C}}$ declines. Based on the simulation results of the proposed optimization algorithm, the energy consumption converges to a minimum value if the holding speed is very close to 0 .

In this section, (FPC) and field operation data are compared with the proposed algorithm under the same trip time (95 $\mathrm{min}$ ), which are illustrated in two case studies. Figure 16 is the simulation result of the proposed algorithm.

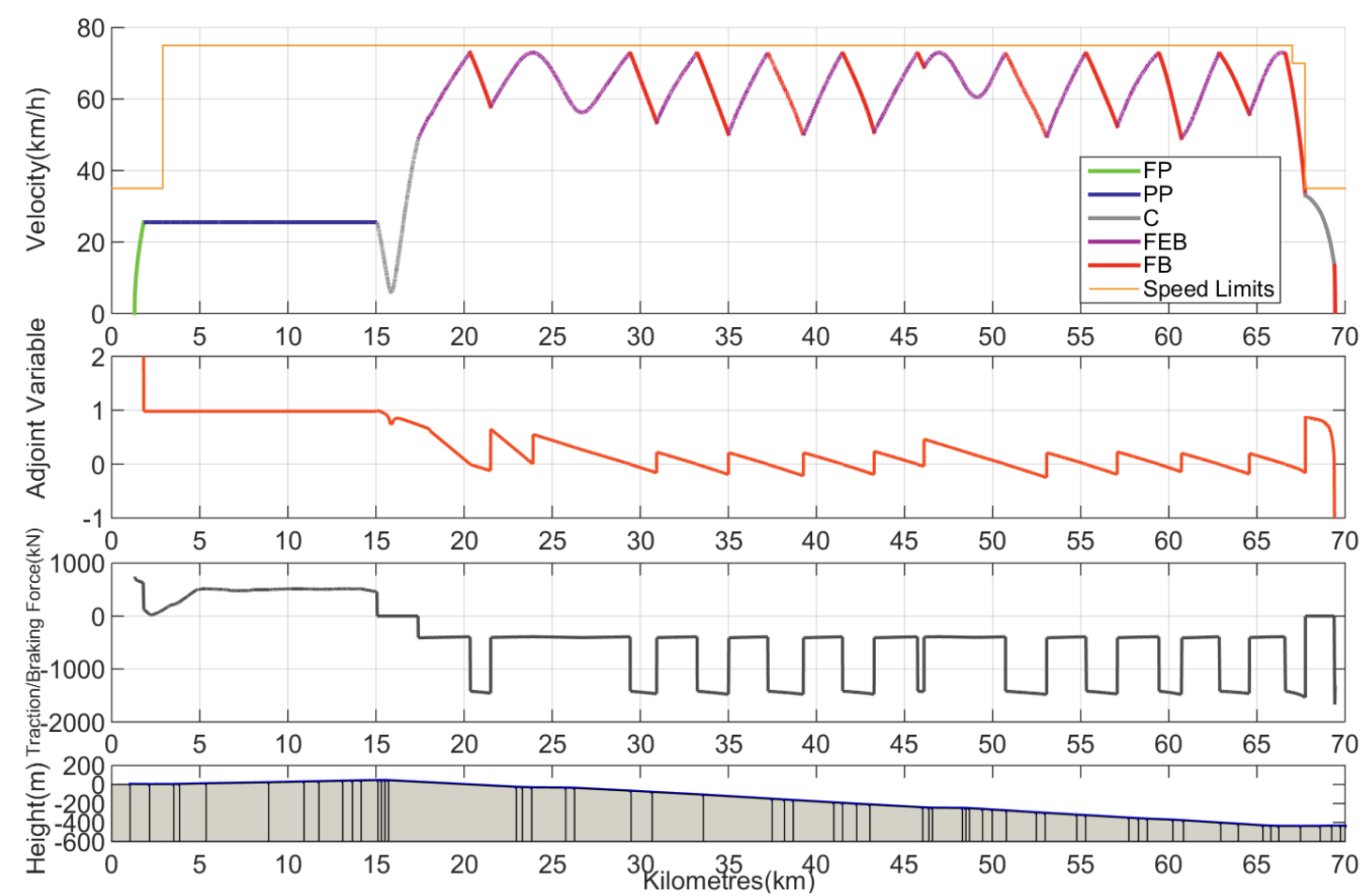

Figure 16. Optimal speed profile of train energy-efficient operation considering LDSD section (trip time: $95 \mathrm{~min})$.

In Figure $16, v_{\mathcal{c}}=25.5 \mathrm{~km} / \mathrm{h}$, the operation time is $5704.20 \mathrm{~s}(\approx 95 \mathrm{~min})$, more details are shown in Table 3. At the origin location, the initial adjoint variable $(\theta)$ is large and it's decreasing quickly with the location of the train increasing. Here, $\theta$ ranges from -1 to 2 . The optimal controls are decided by the value of the adjoint variable. For an example, if $\theta$ declines from 1 to $\alpha \eta_{d} \eta_{t}<\theta<1$, then the optimal control switches from PP to C. Applying coasting before entering the LDSD section makes the freight train operation more energy-efficient because more potential energy is converted to kinetic energy. While the train is running on the LDSD section, FEB and FB are applied alternatively because of the jump of the adjoint variable. The force that varies with location of the train is also shown in Figure 16.

\subsubsection{Fuzzy Predictive Control}

The (FPC) algorithm was designed in [34]. There are two controls for the algorithm: the deviation of the current train speed and target speed, the acceleration of the freight train. They are put into the 
fuzzy logic controller for fuzzy reasoning and optimizing. And the algorithm calculates the control sequences in the future $2-3 \mathrm{~km}$. The simulation result under the given trip time (95 $\mathrm{min}$ ) is presented in Figure 17.
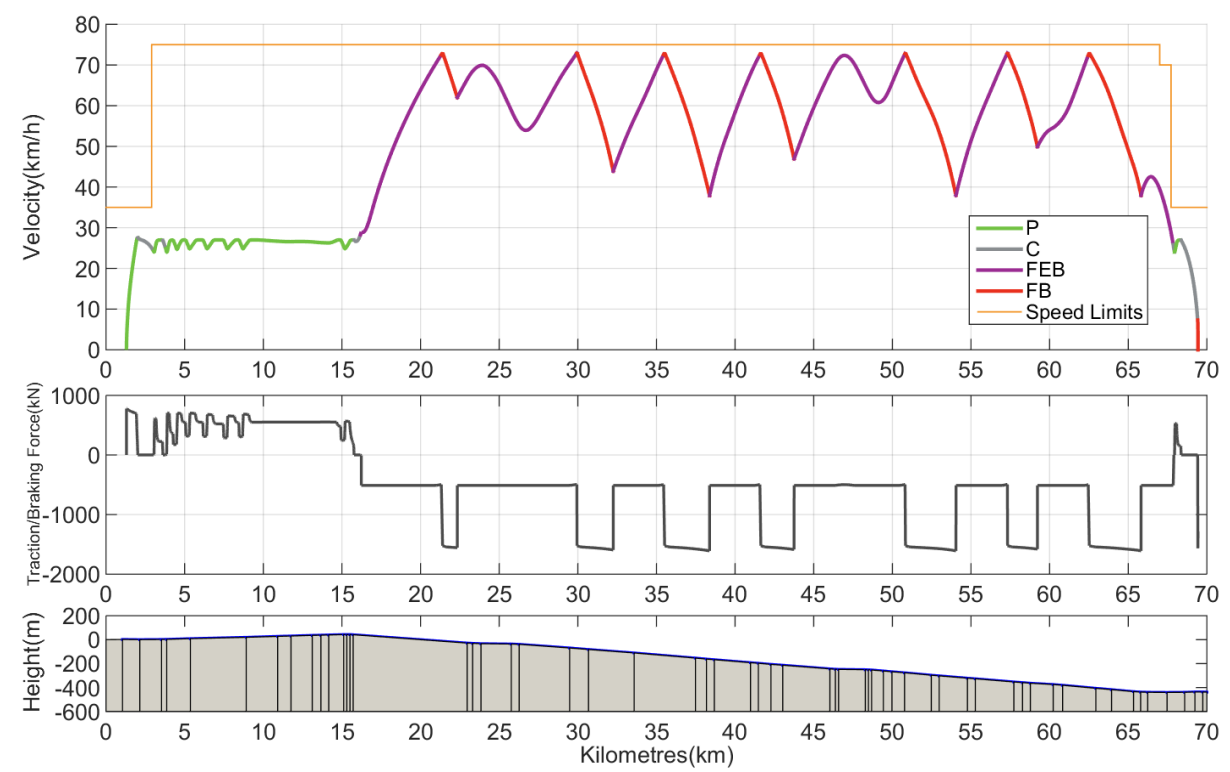

Figure 17. Speed profile of a freight train based on FPC (trip time: $95 \mathrm{~min}$ ).

In Figure 17, $\mathrm{P}=$ Power. The freight train tracks the target speed before entering the LDSD section. The speed rises up while the train is running on the LDSD section. Periodic braking should be applied to prevent the train from exceeding the speed limits. The train energy consumption for traction is $1821.50 \mathrm{kWh}$. The regenerative braking energy $5492.60 \mathrm{kWh}$.

\subsubsection{Field Operation Data}

We obtained the field operation data from Yanqing to Chawu, which consists of the train speed and location. The journey time is $95 \mathrm{~min}$. The speed profile is shown in Figure 18 with $\mathrm{P}=$ Power, $\mathrm{EB}=$ Electrical Braking, $\mathrm{PB}=$ Pneumatic Braking.
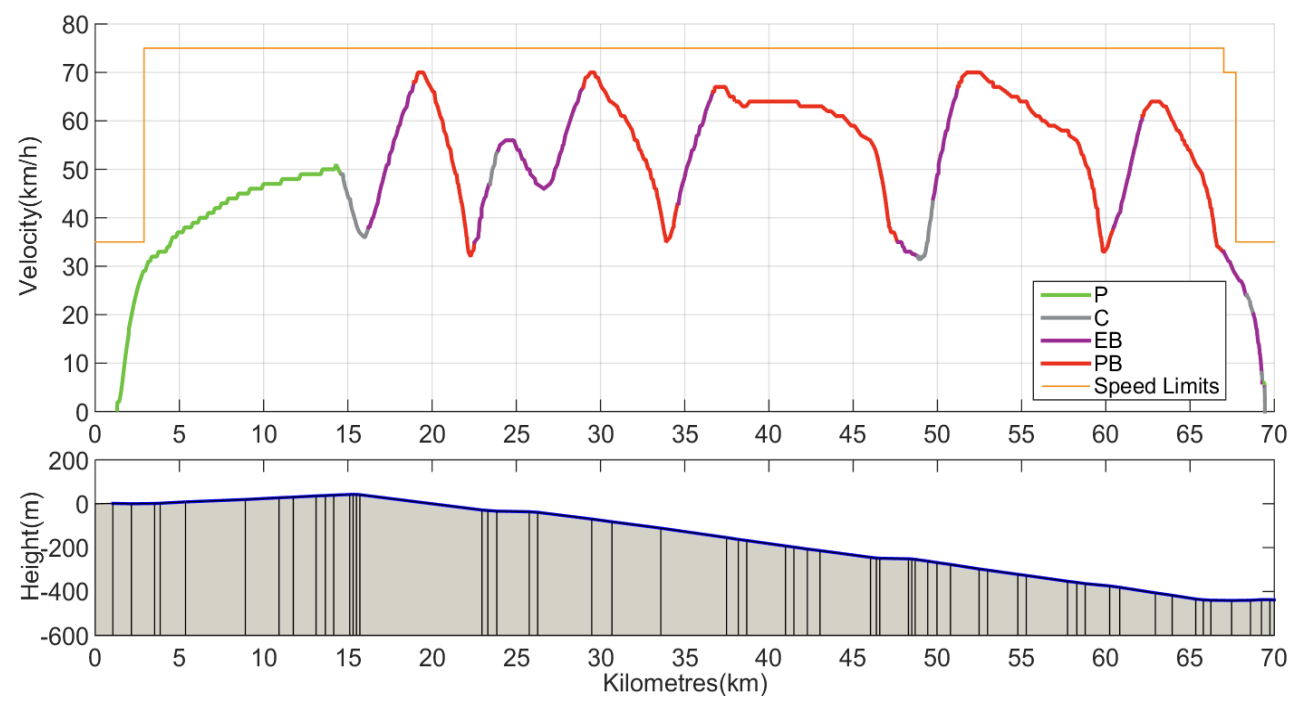

Figure 18. Speed profile of a freight train based on the field operation data (trip time: $95 \mathrm{~min}$ ). 
Besides that, the simulation results of the three strategies (optimal algorithm, FPC and field operation) and the energy-saving ratio comparison of the three strategies are listed in Tables 4 and 5.

Table 4. Simulation results of the three strategies.

\begin{tabular}{cccccc}
\hline Strategies & $\begin{array}{c}\text { Journey Time } \\
(\mathbf{m i n})\end{array}$ & $\boldsymbol{v}_{\boldsymbol{c}} \mathbf{( \mathbf { k m } / \mathbf { h } )}$ & $\begin{array}{c}\text { Energy Consumption } \\
\mathbf{( k W h )}\end{array}$ & $\begin{array}{c}\text { Regenerative Energy } \\
\mathbf{( k W h )}\end{array}$ & $\begin{array}{c}\text { Total Energy Consumption } \\
\mathbf{( k W h )}\end{array}$ \\
\hline Optimal algorithm & 95 & 25.5 & 1701.85 & 5583.39 & -3323.20 \\
FPC & 95 & - & 1821.50 & 5492.60 & -3121.84 \\
Field operation & 95 & - & 1906.50 & 5231.21 & -2801.59 \\
\hline
\end{tabular}

Table 5. Energy-saving ratio comparison of the three strategies.

\begin{tabular}{ccc}
\hline Strategies & FPC (\%) & Field Operation (\%) \\
\hline Optimal algorithm & 6.5 & 18.6 \\
FPC & - & 11.4 \\
\hline
\end{tabular}

The driving strategy is usually determined by the current train running status and the track gradient, curve, speed limit in the future $2-3 \mathrm{~km}$ while a driver is operating the freight train. Meanwhile, excellent driving experience is also significant, which ensures the energy-efficient operation of the train. For an example, an experienced driver will apply coasting before the train enters the LDSD section (see Figure 18). However, the driver operates the train intuitively without calculating the precise traction/braking force and the switching location of the optimal controls. Then $18.6 \%$ and $11.4 \%$ reduction of the train energy consumption can be achieved respectively compared with that of the field operation and FPC. FPC is a local optimization algorithm which predicts the optimal controls in the finite distance ahead of the train. However, the proposed algorithm in this paper is a global optimization algorithm. We can conclude that the optimal algorithm saves more energy than that of FPC (6.5\%).

\section{Conclusions}

In this paper, an analytical solution and numerical algorithm are proposed to solve the energy-efficient operation problem considering the LDSD section issue. A new path constraint-auxiliary reservoir air-filled time constraint is taken into consideration in the optimal control model of the train energy-efficient operation. The adjoint variable is utilized to illustrate the optimal control set and calculate the optimization trajectory of the two adjacent speed holding sections. Analysis of the adjoint variable shows that periodic braking (FEB+FB+FEB) should be applied on the LDSD section, which can effectively reduce the operation time on the LDSD section and save the energy consumption of the entire trip. The numerical algorithm is adopted to calculate the optimization trajectory under the given trip time. Case studies indicate that the proposed algorithm in this paper saves $6.5 \%$ and $18.6 \%$ of the energy consumption comparing with that of FPC and field operation, which has a better performance with respect to energy saving.

The numerical algorithm of the train energy-efficient operation has been implemented in MATLAB. From the perspective of engineering application, the proposed algorithm in this paper could be utilized to calculate the off-line optimal speed trajectory for the driver advisory system. Based on the improved numerical algorithm considering the signal system, temporary speed limit and current train status, the driver advisory system calculates the dynamic optimal speed trajectory and provides the driver dynamic advice about the energy-efficient driving strategies.

Acknowledgments: This work is supported by the National Key Research and Development Plan of China under Grant 2016YFB1200502. 
Author Contributions: Xuan Lin and Qingyuan Wang conceived and designed the numerical algorithm; Xuan Lin programmed the numerical algorithm; Xuan Lin and Qingyuan Wang analyzed the data; Pengfei Sun and Pengling Wang gave very constructive suggestions about the revision of the paper; Xiaoyun Feng provided the line data, train data and field operation data. Xuan Lin wrote the paper.

Conflicts of Interest: The authors declare no conflict of interest.

\section{References}

1. Han, J. China Railway Year Book 2008; China Railway Publishing House: Beijing, China, 2008.

2. Li, L.; Dong, W.; Ji, Y.; Zhang, Z.; Tong, L. Minimal-energy driving strategy for high-speed electric train with hybrid system model. IEEE Trans. Intell. Transp. Syst. 2013, 14, 1642-1653. [CrossRef]

3. Sidelnikov, V. Computation of optimal controls of a railroad locomotive. Proc. State Railw. Res. Inst. 1965, 2, 52-58.

4. Ichikawa, K. Application of optimization theory for bounded state variable problems to the operation of train. Bull. JSME 1968, 11, 857-865. [CrossRef]

5. Milroy, I.P. Aspect of Automatic Train Control. Ph.D. Thesis, Loughborough University, Loughborough, UK, 1980.

6. Asnis, I.A.; Dmitruk, A.V.; Osmolovskii, N.P. Solution of the problem of the energetically optimal control of the motion of a train by the maximum principle. USSR Comput. Math. Math. Phys. 1985, 25, 37-44. [CrossRef]

7. Lee, D.H.; Milroy, I.P.; Tyler, K. Application of Pontryagin's maximum principle to the semi-automatic control of rail vehicles. In Proceedings of the Second Conference on Control Engineering, Newcastle, Australia, 1-3 April 1982.

8. Howlett, P.G.; Pudney, P.J.; Vu, X. Local energy minimization in optimal train control. Automatica 2009, 45, 2692-2698. [CrossRef]

9. Albrecht, A.; Howlett, P.G.; Pudney, P.J.; Vu, X.; Zhou, P. The key principles of optimal train control-Part 1: Formulation of the model, strategies of optimal type, evolutionary lines, location of optimal switching points. Transp. Res. Part B Methodol. 2016, 94, 482-508. [CrossRef]

10. Albrecht, A.; Howlett, P.G.; Pudney, P.J.; Vu, X.; Zhou, P. The key principles of optimal train control—Part 2: Existence of an optimal strategy, the local energy minimization principle, uniqueness, computational techniques. Transp. Res. Part B Methodol. 2016, 94, 509-538. [CrossRef]

11. Howlett, P.G. A new look at the rate of change of energy consumption with respect to journey time on an optimal train journey. Transp. Res. Part B Methodol. 2016, 94, 387-408. [CrossRef]

12. Khmelnitsky, E. On an optimal control problem. IEEE Trans. Autom. Control 2000, 45, 1257-1266. [CrossRef]

13. Golovitcher, I.M.; Iakov, M. Energy efficient control of rail vehicles. In Proceedings of the IEEE International Conference Systems, Man, and Cybernetics, Newark, NJ, USA, 7-10 October 2001.

14. Liu, R.; Golovitcher, I.M. Energy-efficient operation of rail vehicles. Transp. Res. Part A Policy Pract. 2003, 37, 917-932. [CrossRef]

15. Yang, J.; Jia, L.; Lu, S.; Fu, Y.; Ge, J. Energy-efficient speed profile approximation: An optimal switching region-based approach with adaptive resolution. Energies 2016, 9, 762. [CrossRef]

16. Jiaxin, C.; Howlett, P. Application of critical velocities to the minimisation of fuel consumption in the control of trains. Automatica 1992, 28, 165-169. [CrossRef]

17. Jiaxin, C.; Howlett, P. A note on the calculation of optimal strategies for the minimization of fuel consumption in the control of trains. IEEE Trans. Autom. Control 1993, 38, 1730-1734. [CrossRef]

18. Howlett, P.G.; Milroy, I.P.; Pudney, P.J. Energy-efficient train control. Control Eng. Pract. 1994, 2, $193-200$. [CrossRef]

19. Howlett, P.G. Optimal strategies for the control of a train. Automatica 1996, 32, 519-532. [CrossRef]

20. Howlett, P.G.; Cheng, J. Optimal driving strategies for a train on a track with continuously varying gradient. J. Aust. Math. Soc. Ser. B Appl. Math. 1997, 38, 388-410. [CrossRef]

21. Franke, R.; Terwiesch, P.; Meyer, M. An algorithm for the optimal control of the driving of trains. In Proceedings of the IEEE Conference on Decision and Control, Sydney, Australia, 12-15 December 2000.

22. Franke, R.; Meyer, M.; Terwiesch, P. Optimal Control of the Driving of Trains. Automatisierungstechnik 2002, 50, 606-613. [CrossRef] 
23. Ko, H.; Koseki, T.; Miyatake, M. Application of dynamic programming to optimization of running profile of a train. Comput. Railw. IX 2004, 125, 103-112.

24. Kuriyama, M.; Yamamoto, S.; Miyatake, M. Theoretical study on Eco-Driving Technique for an Electric Vehicle with Dynamic Programming. In Proceedings of the IEEE Internatioanl Conference on Electrical Machines and Systems (ICEMS), Incheon, Korea, 10-13 October 2010.

25. Miyatake, M.; Matsuda, K. Energy saving speed and charge/discharge control of a railway vehicle with on-board energy storage by means of an optimization model. IEEJ Trans. Electr. Electron. Eng. 2009, 4, 771-778. [CrossRef]

26. Miyatake, M.; Ko, H. Optimization of train speed profile for minimum energy consumption. IEEJ Trans. Electr. Electron. Eng. 2010, 5, 263-269. [CrossRef]

27. Li, L.; Dong, W.; Ji, Y.; Zhang, Z. An optimal driving strategy for high-speed electric train. J. Control Theory Appl. 2012, 10, 280-286. [CrossRef]

28. Wang, Y.; De Schutter, B.; van den Boom, T.J.J.; Ning, B. Optimal trajectory planning for trains-A pseudospectral method and a mixed integer linear programming approach. Transp. Res. Part C Emerg. Technol. 2013, 29, 97-114. [CrossRef]

29. Wang, P.; Goverde, R.M.P. Multiple-phase train trajectory optimization with signalling and operational constraints. Transp. Res. Part C Emerg. Technol. 2016, 69, 255-275. [CrossRef]

30. Lu, S.; Hillmansen, S.; Ho, T.K.; Roberts, C. Single-train trajectory optimization. IEEE Trans. Intell. Transp. Syst. 2013, 14, 743-750. [CrossRef]

31. Wong, K.K.; Ho, T.K. Dynamic coast control of train movement with genetic algorithm. Int. J. Syst. Sci. 2003, 2, 835-846. [CrossRef]

32. Zhao, N.; Roberts, C.; Hillmansen, S.; Nicholson, G. A multiple train trajectory optimization to minimize energy consumption and delay. IEEE Trans. Intell. Transp. Syst. 2015, 16, 2363-2372. [CrossRef]

33. Huang, Y.; Ma, X.; Su, S.; Tang, T. Optimization of train operation in multiple intersections with multi-population genetic algorithm. Energies 2015, 8, 14311-14329. [CrossRef]

34. Feng, X. On Fuzzy Predictive Control and Its Application in ATO. Ph.D. Thesis, Southwest Jiaotong University, Chengdu, China, 2001.

35. Bai, Y.; Ho, T.; Mao, B.; Ding, Y.; Chen, S. Energy-efficient locomotive operation for Chinese mainline railways by fuzzy predictive control. IEEE Trans. Intell. Transp. Syst. 2014, 15, 938-948. [CrossRef]

36. Kang, X.; Sun, J.; Meng, W. Regulations on Railway Traction Calculation (TB/T1407-201X); National Railway Administration of China: Beijing, China, 2014.

37. Afshari, A.; Specchia, S.; Shabana, A.A.; Caldwell, N. A train air brake force model: Car control unit and numerical results. Proc. Inst. Mech. Eng. Part F J. Rail Rapid Transit 2012, 227, 38-55. [CrossRef]

38. Richard, F.H.; Suresh, P.S.; Raymond, G.V. A survey of the maximum principles for optimal control problems with state constraints. SIAM Rev. 1995, 37, 181-218.

39. Sun, Z.; Wang, C. Security problems of operation heavy haul trains on the long heavy down grade. Railw. Locomot. Car 2009, 29, 4-7.

40. Seierstad, A.; Sydsaeter, K. Sufficient conditions in optimal control theory. Int. Econ. Rev. 1977, 18, 367-391. [CrossRef]

41. Sarosh, N.T.; Robert, L.K. Multi-objective trajectory optimization for electric trains. IEEE Trans. Autom. Control 1979, 24, 888-893.

(C) 2017 by the authors. Licensee MDPI, Basel, Switzerland. This article is an open access article distributed under the terms and conditions of the Creative Commons Attribution (CC BY) license (http:/ / creativecommons.org/licenses/by/4.0/). 